\title{
Impact of Processed Food (Canteen and Oil Wastes) on the Development of Black Soldier Fly (Hermetia illucens) Larvae and Their Gut Microbiome Functions
}

\author{
Thomas Klammsteiner ${ }^{1 *}$, Andreas Walter ${ }^{2}$, Tajda Bogataj', Carina D. Heussler ${ }^{1,3}$, \\ Blaž Stres ${ }^{4,5,6}$, Florian M. Steiner ${ }^{3}$, Birgit C. Schlick-Steiner ${ }^{3}$ and Heribert Insam ${ }^{1}$

\begin{abstract}
${ }^{1}$ Department of Microbiology, University of Innsbruck, Innsbruck, Austria, ${ }^{2}$ Department of Biotechnology and Food Engineering, $\mathrm{MCl}$ - The Entrepreneurial School, Innsbruck, Austria, ${ }^{3}$ Department of Ecology, University of Innsbruck, Innsbruck, Austria, ${ }^{4}$ Department of Animal Science, University of Ljubljana, Ljubljana, Slovenia, ${ }^{5}$ Institute of Sanitary Engineering, University of Ljubljana, Ljubljana, Slovenia, ${ }^{6}$ Faculty of Medicine, University of Ljubljana, Ljubljana, Slovenia
\end{abstract}

\section{OPEN ACCESS}

Edited by:

Leen Van Campenhout, KU Leuven, Belgium

Reviewed by:

Stefano Campanaro,

University of Padua, Italy

Stefanie P. Glaeser,

University of Giessen, Germany

*Correspondence:

Thomas Klammsteiner

thomas.klammsteiner@uibk.ac.at

Specialty section: This article was submitted to

Food Microbiology,

a section of the journal

Frontiers in Microbiology

Received: 19 October 2020

Accepted: 04 January 2021

Published: 21 January 2021

Citation:

Klammsteiner $T$, Walter $A$,

Bogataj T, Heussler CD, Stres B, Steiner FM, Schlick-Steiner BC and Insam H (2021) Impact of Processed

Food (Canteen and Oil Wastes) on the Development of Black Soldier Fly (Hermetia illucens) Larvae and Their

Gut Microbiome Functions.

Front. Microbiol. 12:619112.

do: 10.3389/fmicb.2021.619112
Canteens represent an essential food supply hub for educational institutions, companies, and business parks. Many people in these locations rely on a guaranteed service with consistent quality. It is an ongoing challenge to satisfy the demand for sufficient serving numbers, portion sizes, and menu variations to cover food intolerances and different palates of customers. However, overestimating this demand or fluctuating quality of dishes leads to an inevitable loss of unconsumed food due to leftovers. In this study, the food waste fraction of canteen leftovers was identified as an optimal diet for black soldier fly (Hermetia illucens) larvae based on 50\% higher consumption and $15 \%$ higher waste reduction indices compared with control chicken feed diet. Although the digestibility of food waste was nearly twice as high, the conversion efficiency of ingested and digested chicken feed remains unparalleled (17.9 \pm 0.6 and $37.5 \pm 0.9$ in CFD and $7.9 \pm 0.9$ and $9.6 \pm 1.0$ in FWD, respectively). The oil separator waste fraction, however, inhibited biomass gain by at least $85 \%$ and ultimately led to a larval mortality of up to $96 \%$. In addition to monitoring larval development, we characterized physicochemical properties of pre- and post-process food waste substrates. High-throughput amplicon sequencing identified Firmicutes, Proteobacteria, and Bacteroidota as the most abundant phyla, and Morganella, Acinetobacter, and certain Lactobacillales species were identified as indicator species. By using metagenome imputation, we additionally gained insights into the functional spectrum of gut microbial communities. We anticipate that the results will contribute to the development of decentralized waste-management sites that make use of larvae to process food waste as it has become common practice for biogas plants.

\footnotetext{
Keywords: animal feedstuff, waste valorization, circular economy, metabolism, microbial communities, 16S amplicon sequencing, oil waste, growth parameters
}

\footnotetext{
Abbreviations: ANOVA, analysis of variance; BSF, black soldier fly; BSFL, black soldier fly larvae; CFD, chicken feed diet; COD, chemical oxygen demand; DM, dry matter; FOG, fats, oil, and grease; FWD, food waste diet; NMDS, non-metric multidimensional scaling; OTU, operational taxonomic unit; OWD, oil waste diet; VFA, volatile fatty acids; VS, volatile solids.
} 


\section{INTRODUCTION}

With a more fast-moving society in industrialized countries, amounts of food waste in these countries as large as the total net food production of sub-Saharan Africa come along (FAO, 2011). In addition, a recent study by Verma et al. (2020) indicates that the worldwide food waste might be even twice as high than previous statistics published by the Food and Agriculture Organization of the United Nations FAO (2011). In contrast to food loss that occurs early in the food supply chain due to a decrease in quality and improper handling, the extent of food waste originates in the consumers' values, behavior, and attitude (Principato, 2018). This mindset is also reflected by food waste accumulating in food service outlets such as canteens, cafeterias, and buffets. Canteens represent an important source of food waste in hubs of human productivity and many parameters influencing the extent of leftovers have been identified in the past: a lack of flexibility in adapting to consumer preferences, excessive portion sizes, displeasing consumption settings, and time constraints for lunch breaks can be factors for leaving behind food (Boschini et al., 2020).

As a generalist species, the black soldier fly (BSF; Hermetia illucens, Diptera: Stratiomyidae) is frequently used as decomposer of organic wastes, ranging from kitchen waste (Nguyen et al., 2015) and manure (Sheppard et al., 1994) to human excreta (Banks et al., 2014). The application of BSF larvae (BSFL) can range from small-scale lab populations (Nakamura et al., 2016) and household rearing systems (Klammsteiner et al., 2020c) to large-scale industrial rearing operations (Wynants et al., 2018). Food waste is heterogenous in its composition. Elevated salt $(\mathrm{NaCl})$ content in meals is often discussed because of its adverse effect on human health (e.g., high blood pressure) and can account for up to $1.2 \%$ in hot meals and sandwiches handed out in canteens (Rasmussen et al., 2010). Cho et al. (2020) concluded that BSFL are suitable to treat food waste with even higher salt concentrations, since a significant inhibition of biomass gain and pupation was only observed at concentrations $>3 \%$.

The larval gut microbiome is considered essential for efficient food conversion by BSFL. In general, results from microbiome studies on BSFL can be challenging to compare due to variations in biotic and abiotic factors during the execution of experiments (De Smet et al., 2018; Bosch et al., 2020). Recent studies suggest that, although the larval gut microbiome does not remain unaffected when exposed to various biogenic wastes, a versatile core community of bacteria may contribute to the digestion of these substrates (Wynants et al., 2018; Zhan et al., 2019; Klammsteiner et al., 2020b). Although the BSFL gut microbiome has been shown to adapt to changing diets, the overlap between the gut residing communities and communities found in the respective diets is often low (Wynants et al., 2018). Little is known about the microbial metabolism taking place in the larval guts, since past studies have mostly focused on clarifying phylogenetic dynamics in relation to larval diet. Detailed investigations of metabolic processes taking place in larvae and attempts to exploit them for biotechnological applications are sparse (Lee et al., 2018; Song et al., 2018; Zhan et al., 2019). Tools imputing metagenomes to $16 \mathrm{~S}$ rRNA gene-based amplicon data such as
PICRUSt (Langille et al., 2013) or Tax4Fun (Aßhauer et al., 2015) have been used to provide insights into functional genes. Although originally developed for a human microbiome context, the reference databases are continuously growing also for other animals and environments (Breitwieser et al., 2017; Kostanjšek et al., 2019). To comply with the widespread use of amplicon sequencing, both platforms have recently been updated to followup versions (Douglas et al., 2020; Wemheuer et al., 2020). Imputation methods have also become a valuable tool to gain insights in metabolic processes of insects (Chen et al., 2016) and to assess the effect of insect-based diets on livestock such as poultry (Borrelli et al., 2017). In addition, they could provide an efficient approach to screen prokaryotic communities for novel bioactive compounds and find new means for the degradation of xenobiotics as has recently been investigated in marine sponges (Steinert et al., 2019).

In this study, we determined the effect of two major organic-waste fractions occurring in canteens (food waste from leftovers and oil waste from oil separators) on BSFL growth, the bacterial gut biota, and its functional makeup. The larval diet was changed in the experiments from the chicken feed diet (CFD) to either the food (FWD) or oil waste diet (OWD) 6 days after larvae had hatched. Guts were extracted at multiple time points to determine changes in microbial-community composition and function during their development. Finally, larval growth, waste degradation, and developmental time were documented, and meaningful process indices were derived from these data. We hypothesized that, due to its high nutritional value, canteen food waste represents a convenient substrate for BSFL rearing but presumed that it would induce shifts in gut microbiome composition due to varying nutrient patterns. Moreover, we considered that diet-induced shifts in the larval gut microbiota could provide an opportunity to identify diet-specific microbial biomarkers.

\section{MATERIALS AND METHODS}

\section{Source of Black Soldier Fly Larvae and Colony Maintenance}

Six-day old larvae were obtained from a bench-scale BSF colony at the Department of Microbiology (University of Innsbruck, Austria). Adequate and stable environmental conditions of $27^{\circ} \mathrm{C}$, $60 \%$ relative humidity, and a light:dark photoperiod of $16: 8 \mathrm{~h}$ using LED panels as described in Heussler et al. (2018) were created in a Fitotron SGC 120 (Weiss Technik, United Kingdom) climate chamber. Larvae, pupae, and adults were held in separate containers, and the density of individuals was manually controlled. An ad libitum amount of ground chicken feed diet (CFD; Grünes LegeKorn Premium, Unser Lagerhaus, Austria) mixed with water $(40: 60 \mathrm{w} / \mathrm{v})$ was used for colony maintenance.

\section{Experiment Preparation and Diets}

Six days after hatching, 200 manually counted larvae were each transferred to four replicate boxes per diet, adding up to a total of 2,400 larvae equally distributed over twelve separate boxes with a density of 1.2 larvae $\mathrm{cm}^{-2}$. The BSFL were kept under the 
same conditions as described for the general colony maintenance. Sterilized and dried pine humus ( $20 \mathrm{~g}$ ) was added to each box as litter for humidity regulation. The diets were defined as OWD, FWD, and CFD analogous to colony maintenance as control diet. OWD and FWD were obtained from a local canteen on a single day (DMS: $47^{\circ} 15^{\prime} 50.9^{\prime \prime} \mathrm{N}, 11^{\circ} 20^{\prime} 37.4^{\prime \prime} \mathrm{E}$ ). For OWD, the upper layers from the content of an oil separator collection container were collected and manually homogenized. Fresh canteen waste for the FWD was shredded with a Vitamix TNC ${ }^{\circledR}$ electric blender (Vitamix, Olmsted Falls, OH, United States), thereby reducing the size of food residues and homogenizing its components. The diets were stored at $-20^{\circ} \mathrm{C}$ until the start of the experiment. Feeding took place every second day for a maximum of 18 days $\left(\mathrm{d}_{0-18}\right)$, resulting in a maximum of ten feeding events per diet. After 20 days $\left(d_{20}\right)$, the experiment was terminated. The amount of substrate to administer per larva and day was calculated using the content of organics in $100 \mathrm{mg} \mathrm{CFD} \mathrm{larva}^{-1}$ as reference. OWD and FWD were thereby added in amounts of 70 and $170 \mathrm{mg} \mathrm{larva}^{-1}$ day $^{-1}$, respectively. Water and organic content were determined gravimetrically by measuring the loss of mass after drying the samples in a drying oven (UF110, Memmert, Schwabach, Germany) at $105^{\circ} \mathrm{C}$ for $24 \mathrm{~h}$ and subsequent incineration in a muffle furnace (CWF 1000, Carbolite, Neuhausen, Germany) at $550^{\circ} \mathrm{C}$ for $5 \mathrm{~h}$. Substrate-water content was adapted to the moistest substrate (FWD) by adding 70 and $100 \mathrm{mg}$ of water to CFD and OWD, respectively. Thereby, all three diets contained the same amount of water and organics.

\section{Sample Collection and Preparation and Processing}

Sampling followed the scheme illustrated in Figure $\mathbf{1}$ and samples were stored at $-20^{\circ} \mathrm{C}$ if not immediately processed. A climate room operating at $4^{\circ} \mathrm{C}$ was used to slowly thaw frozen samples. One larval sample consisted of ten randomly

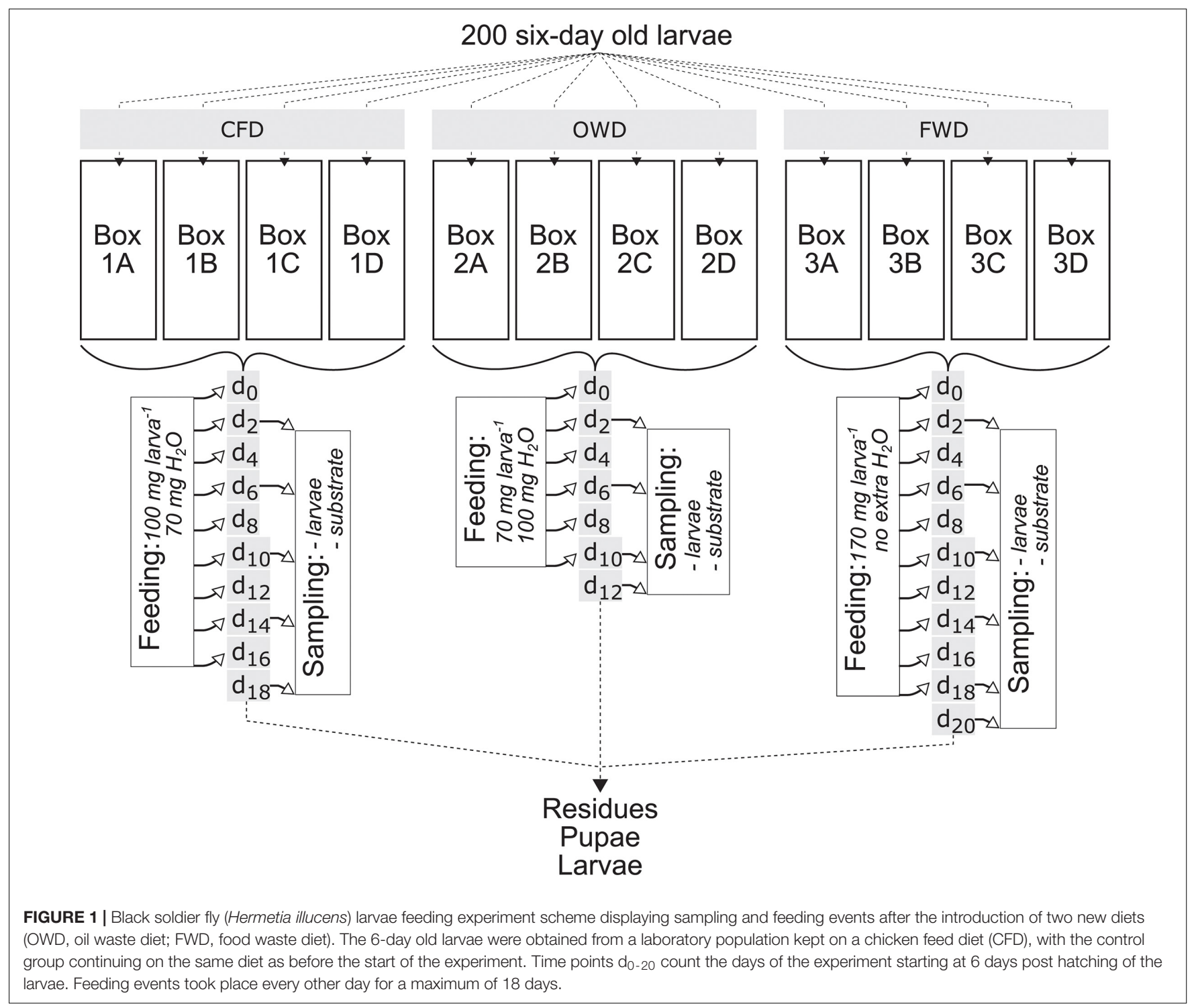


collected larvae, of which five were used for determination of dry matter (DM) and volatile solids (VS), and another five were subjected to gut extraction. Fresh substrates and substrate residues were both physicochemically characterized prior and after the experiment, respectively. To prepare samples for the quantification of oxidizable organics [chemical oxygen demand (COD)], ammonium, total protein and reducing sugars, $10 \mathrm{~g}$ of

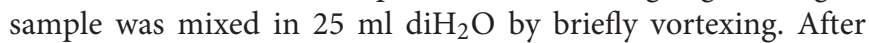
incubation at room temperature for $30 \mathrm{~min}$, the mixtures were centrifuged at $12,000 \times g$ for $30 \mathrm{~min}$ and subsequently filtered (MN 615 1/4 150 mm, Macherey-Nagel, Düren, Germany). The filtrate was used for further analyses.

\section{Physicochemical Analyses Total Solids, C/N Ratio, Volatile Solids, and Fatty Acids}

Total solids were determined gravimetrically after drying the samples at $105^{\circ} \mathrm{C}$ for $24 \mathrm{~h}$. The difference in fresh and dry mass was determined as water content. Volatile solids were inferred from the weight difference before and after incinerating the dry samples in a muffle furnace at $550^{\circ} \mathrm{C}$ for $5 \mathrm{~h}$. Samples dried at $105^{\circ} \mathrm{C}$ for $24 \mathrm{~h}$ were finely ground using a pestle and mortar to quantify the $\mathrm{C} / \mathrm{N}$ content using an elemental analyzer (TruSpec CHN Elemental Determinator, Leco, St. Joseph, MI, United States) by following the manufacturer's protocol. For the determination of volatile fatty acids, one g per sample was mixed into $1 \mathrm{ml}$ sterile water and centrifuged for $15 \mathrm{~min}$ at $15,000 \times g$. Vials for high performance liquid chromatography were filled with the liquid supernatant and used for analysis following Wagner et al. (2017).

\section{Chemical Oxygen Demand, Ammonium, Total Protein Content, and $\mathrm{pH}$}

After filtration (section "Sample Collection and Preparation and Processing"), the samples were diluted in $\mathrm{diH}_{2} \mathrm{O}$ following the protocol enclosed in the quick test kits Nanocolor COD 1500 and Nanocolor Ammonium 50 (Macherey-Nagel, Düren, Germany), respectively, and transferred in the cuvettes. The cuvettes were incubated at $160^{\circ} \mathrm{C}$ for $30 \mathrm{~min}$ (Nanocolor Vario
HC, Macherey-Nagel, Düren, Germany) and photometrically measured (Nanocolor UV/VIS, Macherey-Nagel, Düren, Germany). The Lowry protein assay following Noble and Bailey (2009) was used to determine the total protein content in sample filtrates. For the $\mathrm{pH}$ measurement, $10 \mathrm{~g}$ of substrate were mixed in $25 \mathrm{ml} \mathrm{diH} \mathrm{H}_{2} \mathrm{O}$ and briefly vortexed. After $60 \mathrm{~min}$ incubation at room temperature the $\mathrm{pH}$ was determined with a $774 \mathrm{pH}$ Meter (Metrohm, Herisau, Switzerland).

\section{Statistical Analysis of Larval Development and Physicochemical Measurements}

Growth rate, consumption index, and approximate digestibility were calculated based on Waldbauer (1968). Substrate reduction and the waste reduction index were determined as described by Jucker et al. (2020) and efficiency of conversion of the ingested and digested food was calculated as in Medrano and Gall (1976). Applied formulae are listed in Table 1. Statistical analyses were conducted in $\mathrm{R}$ ( $\mathrm{R}$ Core Team, 2018). Processing of principal component analysis results and hierarchical clustering were conducted using the factoextra package (Kassambara and Mundt, 2020). All figures were created using ggplot2 (Wickham, 2016).

\section{Harvesting of Larval Guts}

After thawing, larvae were surface sterilized by briefly washing them in a Petri dish containing 70\% EtOH. After the EtOH evaporated, a few $\mathrm{mm}$ were cut off from the anterior part of the larvae using a sterile scalpel and the gut was pulled out from this incision. From each time point $\times$ diet, $0.05 \mathrm{~g}$ guts were pooled from five larvae and transferred to a bead tube using sterile tweezers (Klammsteiner et al., 2020b).

\section{DNA Extraction and 16S rRNA Gene Amplicon Sequencing}

For DNA extraction from fresh substrates, $10 \mathrm{~g}$ of sampling material was mixed with $25 \mathrm{ml}$ deionized water. After vortexing and subsequent incubation for $30 \mathrm{~min}$ at room temperature, the samples were shaken for $30 \mathrm{~min}$ at $120 \mathrm{rpm}$ (Controlled Environment Incubator Shaker, New Brunswick Scientific,

TABLE 1 | Larval growth and degradation parameters based on data collected during the feeding trials $(n=4)$.

\begin{tabular}{|c|c|c|c|c|}
\hline & CFD & FWD & OWD & \\
\hline & mean \pm SD & mean \pm SD & mean \pm SD & Formulae \\
\hline Growth rate $\left[\mathrm{mg} \mathrm{d}^{-1}\right]$ & $9.0 \pm 0.4^{\mathrm{a}}$ & $8.2 \pm 0.8^{a}$ & $1.1 \pm 1.1^{b}$ & $G R=\frac{L_{\text {end }}-L_{\text {initial }}}{T}$ \\
\hline Substrate reduction [\%] & $65.6 \pm 0.2^{\mathrm{a}}$ & $85.3 \pm 0.7^{b}$ & $2.4 \pm 3.7^{c}$ & $\mathrm{SR}=\frac{l-R}{l} \times 100$ \\
\hline Consumption index & $96.9 \pm 1.8^{\mathrm{a}}$ & $156.2 \pm 3.9^{b}$ & $17.5 \pm 27.8^{\mathrm{C}}$ & $C l=\frac{E}{T \times A}$ \\
\hline Waste reduction index & $3.7 \pm 0.0^{a}$ & $4.3 \pm 0.0^{b}$ & $0.2 \pm 0.3^{c}$ & $\begin{array}{l}D=\frac{I-R}{T} \\
W R I=\frac{D}{T} \times 100\end{array}$ \\
\hline Approximate digestibility [\%] & $47.7 \pm 0.5^{a}$ & $82.7 \pm 0.9^{b}$ & - & $A D=\frac{E-R}{E} \times 100$ \\
\hline Efficiency of conversion of ingested food [\%] & $17.9 \pm 0.6^{a}$ & $7.9 \pm 0.9^{b}$ & - & $E C l=\frac{B}{E} \times 100$ \\
\hline Efficiency of conversion of digested food [\%] & $37.5 \pm 0.9^{a}$ & $9.6 \pm 1.0^{b}$ & - & $E C D=\frac{B}{E-R} \times 100$ \\
\hline
\end{tabular}

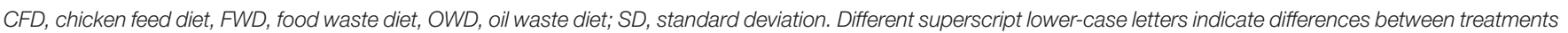

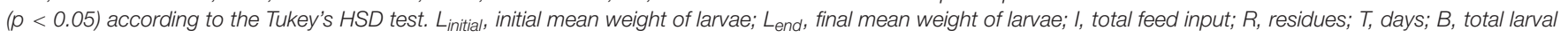
biomass gained; $A$, mean fresh weight during feeding period; $E$, total feed ingested. 
United States) before filtering them through folded filters (MN 615 1/4 150 mm, Macherey-Nagel, Düren, Germany). The DNA of substrate filtrates and guts was extracted following the manual of the used kit (NucleoSpin Soil, Macherey-Nagel, Düren, Germany). Quality and quantity of extracts were assessed via agarose gel electrophoresis and spectrophotometry (NanoDrop 2000c, Thermo Fisher Scientific, Waltham, MA, United States), respectively. Biological replicates of gut samples from three different boxes per treatment were collected and prepared at each sampling time point. Amplicon sequencing including a two-step PCR library preparation using a Nextera Index Kit, purification, pooling, and demultiplexing was performed by Microsynth (Balgach, Switzerland) on a Illumina MiSeq following the $2 \times 250$ bp paired-end approach. The universal bacterial and archaeal primer set 515f/806r (GTGCCAGCMGCCGCGGTAA/GGACTACHVGGGTWTCT AAT) was used to amplify the V4 region on the $16 \mathrm{~S}$ rRNA gene (Caporaso et al., 2011). The provided reads were trimmed from adaptors and primers by the sequencing provider.

\section{Data Analysis of Sequencing Data}

Trimmed raw sequences were analyzed using mothur v.1.44.1 (Schloss et al., 2009). In the initial screening, ambiguous bases, homopolymers longer than eight and sequences $>275$ bases were removed. The SILVA database v.138 (Quast et al., 2013) was used as reference for alignment and classification steps. Potentially chimeric sequences were identified and subsequently removed using the vsearch algorithm. Sequences assigned to chloroplast, mitochondrial, archaeal, eukaryotic, and unknown lineages were filtered from the data. Downstream analyses were carried out in two comparative approaches: (I) binning at $97 \%$ sequence similarity and (II) identification of unique amplicon sequence variants (ASV). For data normalization, samples from both approaches were subsampled to (a) smallest sample size, (b) 15,000 sequences, and (c) 80,000 sequences. Distance matrices based on Bray-Curtis dissimilarity were calculated from the six abundance tables and pairwise comparison of matrices via Mantel test (Pearson correlation, 1000 permutations) was carried out to ensure stability of subsequent analyses (Supplementary Figure 1). The sampling effort was evaluated based on rarefaction curves (Supplementary Figure 2). Pairwise comparison of $\alpha$-diversity (Shannon index; H') in samples grouped by diet and time was conducted using analysis of variance (ANOVA), pairwise $t$-test (including Bonferroni correction), and Tukey's honestly significant difference (HSD) test (Supplementary Table 3). Bartlett's test was applied to test for homogeneity of variances. Non-metric multidimensional scaling (NMDS) based on Bray-Curtis dissimilarity was used as $\beta$-diversity measure on filtered gut microbiome data (minimum prevalence of four in at least $10 \%$ of samples to remove overly sparse OTUs). Overall impact of the variables diet and time on microbial communities was computed by PERMANOVA (Bray-Curtis dissimilarity, 1000 permutations). All statistical tests were conducted using vegan v.2.5.6 (Oksanen et al., 2018). Pielou's evenness (J) was calculated as $\mathrm{H}^{\prime} / \log ($ species number). Congruent OTUs from linear discriminant analysis (LDA) effect size (Segata et al., 2011) implemented in mothur and multi-level pattern analysis as part of the $\mathrm{R}$ indicspecies package (De Cáceres and Legendre, 2009) were considered as indicator species.

Imputation of bacterial metabolic pathways was conducted using version 1.1 .5 of the Tax4Fun2 $\mathrm{R}$ package and the KEGG database (Kanehisa and Goto, 2000) following the standard SOP (Wemheuer et al., 2020). Reference sequences were preclustered at $100 \%$ identity. All figures were created in R using the ggplot 2 package (Wickham, 2016).

\section{RESULTS}

\section{Maturation of Larvae and Dynamics of Waste Degradation}

The feeding experiment was observed for a maximum timespan of 20 days $\left(\mathrm{d}_{20}\right)$ for FWD as the most prolific diet, resulting in 26-day-old larvae at the end of the experiment (Figure 1). The dietary treatments were terminated when all larvae had either pupated or died (CFD at $\mathrm{d}_{18}$, OWD at $\mathrm{d}_{12}$ ). Larvae on CFD and FWD both exhibited similar growth progress, with larvae offered FWD achieving a slightly higher biomass throughout the experiment (Figure 2A). However, fresh weights in the last sample were $5 \%$ lower in FWD, and biomass peaks were reached 2-6 days before the transition to pupal stage in CFD and FWD, respectively. After reaching the biomass peak at $206 \pm 9 \mathrm{mg} \mathrm{larva}^{-1}$ on $\mathrm{d}_{14}$ and continuing their development toward pupae, biomass of FWD-fed larvae significantly decreased by $20 \pm 3 \%$ until $d_{20}(p<0.05$, ANOVA; decrease of $7 \pm 5 \%$ in CFD). Drying and subsequent incineration of larvae additionally revealed similar dry matter (35-37\%; of which $32-36 \%$ were volatile solids) and water contents (63-65\%) in relation to fresh weight (Table 2). However, BSFL fed with OWD were strongly inhibited in their growth and further restricted by a mortality of $96 \pm 2 \%$, which ultimately led to the termination of this dietary treatment at $\mathrm{d}_{12}$. The lethal effect became evident after $\mathrm{d}_{5}$ and surviving larvae exponentially decreased until $\mathrm{d}_{12}$. No larva exposed to OWD transitioned to the pupal stage. The endpoint biomass per larva reared on this diet added up to $21.5 \pm 1.1 \mathrm{mg}$ and was thus $87 \%$ below the weight of larvae reared on CFD and FWD.

With an average developmental time of $22 \pm 3$ days, BSFL raised on FWD developed slightly slower and with higher variability than larvae in the control group ( $21 \pm 2$ days). On CFD and FWD, first pupae appeared at $\mathrm{d}_{12}$ with a significantly higher initial biomass per pupa $(p<0.001)$ in FWD (Figure 2B). While pupae from FWD showed constant fresh weights of $169 \pm 2 \mathrm{mg}$ pupa $^{-1}$ irrespective of the sampling event, pupae raised on CFD gradually increased from $116 \pm 14 \mathrm{mg} \mathrm{pupa}^{-1}$ on $\mathrm{d}_{12}$ to $152 \pm 2 \mathrm{mg} \mathrm{pupa}^{-1}$ in the experiment's last sampling event on $\mathrm{d}_{18}$. In FWD, the last pupae were harvested on $\mathrm{d}_{20}$ and exhibited the largest variation in weight. Pupation in CFD reached its peak on $\mathrm{d}_{16}$ when biomass was highest (154 $\left.\pm 5 \mathrm{mg} \mathrm{pupa}^{-1}\right)$, resulting in around $40 \%$ of larva transitioning to pupal stage in this time point.

In both CFD and FWD, larvae showed comparable growth rates (Table 1) with approx. $8.5 \mathrm{mg}$ biomass gain per day. Although substrate reduction, consumption and waste reduction 

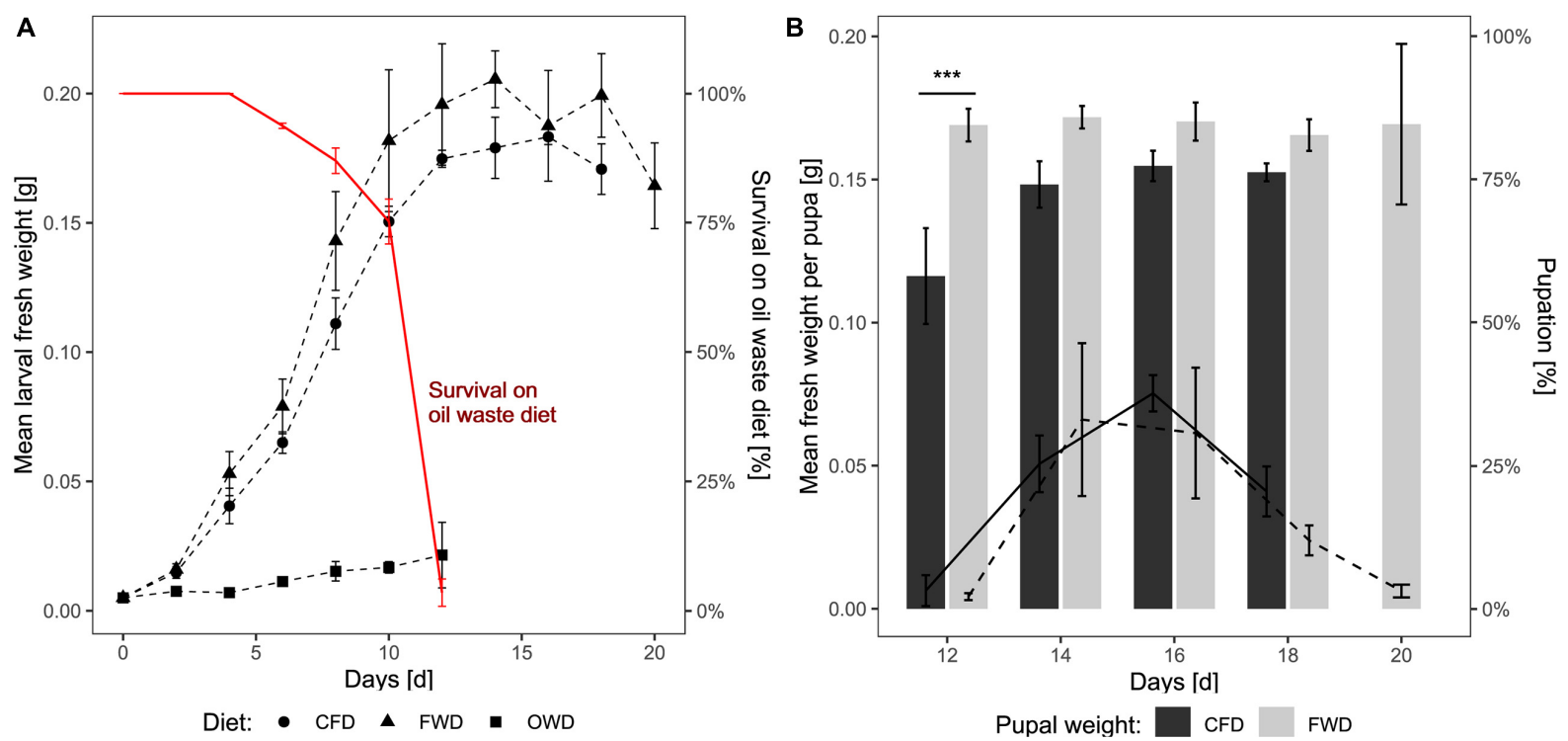

Pupal weight: $\square$ CFD $\square$ FWD

Relative pupation: - CFD - - FWD

C

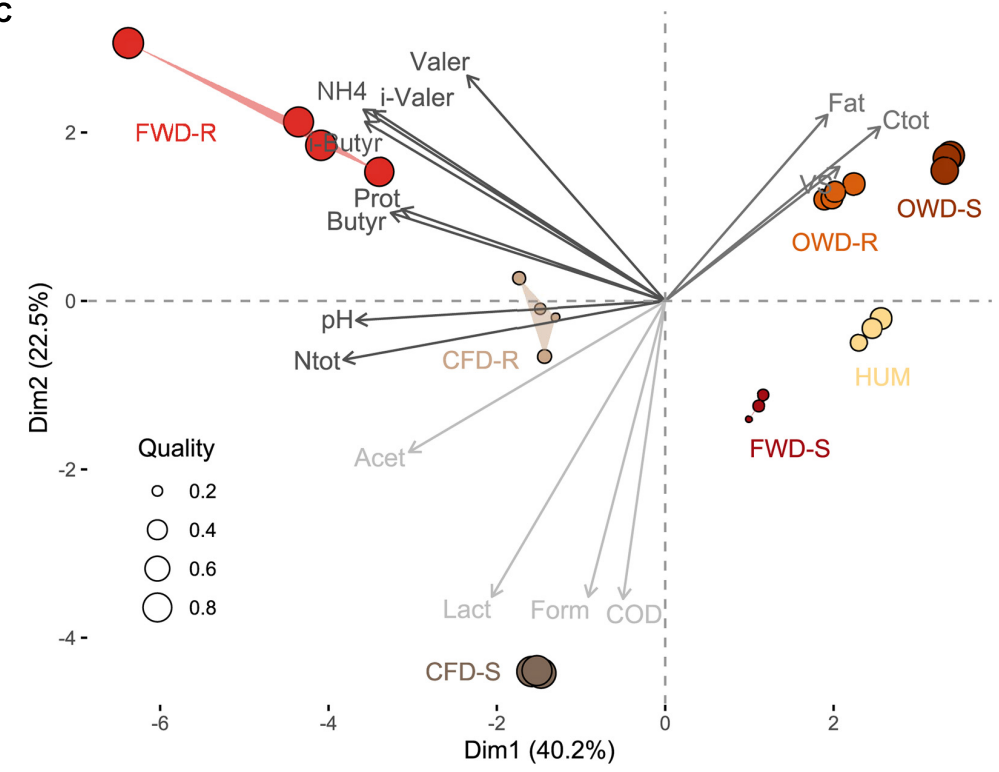

D

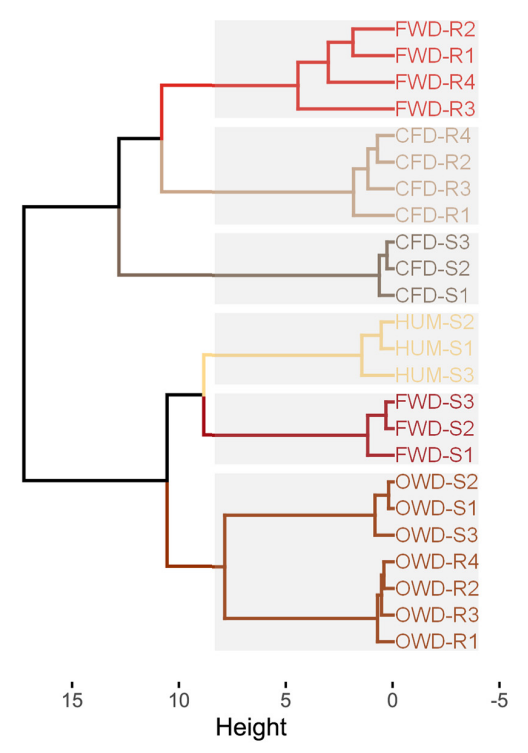

FIGURE 2 | Performance of black soldier fly larvae, pupae, and their three diets. (A) Dotted lines represent the impact of diet on the increase of larval fresh weight $(n=4)$. The red line highlights the survival of larvae on OWD. (B) Bars indicate the fresh weight of pupae in relation to their time of harvest. The lines show the fraction of larvae that transitioned to pupal stage in each sampling time point. Larvae fed with OWD did not reach pupal stage and are not shown in the graph ( $n=4)$. (C) Principal component analysis based on physicochemical properties of fresh substrates (-S) and substrate residues (-R). The three clusters of variables represented by the arrows were determined by k-means clustering and are distinguished by different shades of gray (fresh: $n=3$; residues: $n=4$ ). (D) Hierarchical clustering of sample replicates. Gray backgrounds represent sample clusters determined by the gap statistics method. CFD, chicken feed diet; FWD, food waste diet; OWD, oil waste diet; HUM = pine humus; Prot, proteins; Ntot, total nitrogen; Ctot, total carbon; VS, volatile solids; COD, chemical oxygen demand; (i-)Valer, (i-)valerate; (i-)Butyr, i-butyrate; Form, formate; Lact, lactate; Acet, acetate. ${ }^{\star \star \star} p<0.001$.

indices as well as approximate digestibility were significantly higher in FWD, the efficiency of larvae to convert ingested and digested food were more than two and four times higher in the CFD control diet, respectively. Larvae raised on OWD exhibited negligible substrate reduction. The consumption and waste reduction indices indicated insignificant degradation of the diet fed.

\section{Physicochemical Characterization of Substrates}

Principal component analysis was conducted on physicochemical data from pre-process fresh substrates used as diet (CFD-S, FWD-S, OWD-S) and in post-process substrate residues (CFD-R, FWD-R, OWD-R). The two principal components in Figure 2C explain a combined variance of $62.7 \%$ in the data. The 
TABLE 2 | Endpoint characterization of larval and pupal biomass $(n=4)$.

\begin{tabular}{|c|c|c|c|c|}
\hline & & CFD & FWD & OWD \\
\hline & & mean \pm SD & mean \pm SD & mean \pm SD \\
\hline \multirow[t]{6}{*}{ Larvae } & Fresh weight* $[\mathrm{g}]$ & $0.171 \pm 0.008^{a}$ & $0.164 \pm 0.014^{a}$ & $0.022 \pm 0.011^{b}$ \\
\hline & Water content* $[\mathrm{g}]$ & $0.108 \pm 0.006^{a}$ & $0.107 \pm 0.012^{a}$ & $0.014 \pm 0.006^{b}$ \\
\hline & Dry weight* [g] & $0.063 \pm 0.003^{a}$ & $0.057 \pm 0.001^{a}$ & $0.008 \pm 0.005^{b}$ \\
\hline & Volatile solids* [g] & $0.055 \pm 0.003^{a}$ & $0.054 \pm 0.001^{a}$ & $0.008 \pm 0.005^{b}$ \\
\hline & Mortality [\%] & $0^{b}$ & $0^{b}$ & $96 \pm 2^{a}$ \\
\hline & Developmental time [d] & $21 \pm 2$ & $22 \pm 3$ & 0 \\
\hline \multirow[t]{5}{*}{ Pupae } & Pupation rate [\%] & $87 \pm 7^{a}$ & $80 \pm 9^{a}$ & 0 \\
\hline & Fresh weight $^{+}[\mathrm{g}]$ & $0.153 \pm 0.003^{a}$ & $0.169 \pm 0.023^{a}$ & - \\
\hline & Water content ${ }^{+}[\mathrm{g}]$ & $0.096 \pm 0.001^{a}$ & $0.107 \pm 0.014^{a}$ & - \\
\hline & Dry weight+ [g] & $0.057 \pm 0.001^{a}$ & $0.062 \pm 0.009^{a}$ & - \\
\hline & Volatile solids ${ }^{+}[\mathrm{g}]$ & $0.050 \pm 0.001^{a}$ & $0.060 \pm 0.009^{a}$ & - \\
\hline
\end{tabular}

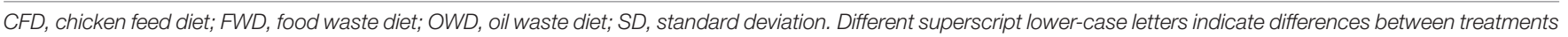
( $p<0.05$ ) according to the Tukey's HSD test.

*at last sampling time point $\left(d_{20}\right)$.

+ after harvesting of pupae.

15 most influential parameters represented by the arrows were aggregated in three groups by k-means clustering to clarify the coordination of samples. Increased $\mathrm{NH}_{4}$ (20- and 30-fold in CFD-R and FWD-R, respectively) and protein (10-fold in all residues) contents strongly contributed in distinguishing pre- from post-process substrates (Supplementary Table 1). Differences between fresh and residue samples from OWD were less evident due to similar physicochemical characteristics featuring a high fat, $\mathrm{C}_{\text {tot }}$, and volatile solid content, therefore, both groups sharing a similar coordination. The fresh control diet CFD-S stands out from the other diets due to its elevated lactate and formate content and higher chemical oxygen demand (COD). After processing by BSFL, the residues of the control diet (CFD-R) deviated from their initial properties and shared more similarities with FWD-R, being better represented by a higher $\mathrm{pH}$ and $\mathrm{N}_{\text {total }}$ content. While FWD-S featured a comparatively scarce VFA profile (Supplementary Table 2) and a weaker fit by the principal components, the residues from this diet (FWD-R) were characterized by high protein, $\mathrm{NH}_{4}$, (i-)valerate, and (i-)butyrate concentrations. The VFAs together with nitrogen compounds (protein, $\mathrm{NH}_{4}, \mathrm{~N}_{\text {total }}$ ) were most influential for the variation in the data. The sterilized and dried pine humus (HUM) used as neutral medium for moisture regulation during the feeding experiment contained negligible concentrations of VFAs, fats and nitrogen compounds. Relationships among groups of pre- and postprocess substrates were further determined by hierarchical clustering (Figure 2D). Gap-statistics analysis (cut-off at $k=0.61$ ) identified an optimal number of six clusters (from seven sample groups) represented by gray rectangles underlaying the dendrogram, summarizing the groups of fresh and residual OWD samples in one group.

\section{Analysis of the Substrate Microbiota}

After passing through quality control and filtering steps, a total of $7.01 \times 10^{5}$ reads from nine fresh substrate samples (three biological replicates per substrate) were used for downstream analysis. ANOVA and PERMANOVA of fresh substrate-derived microbial communities indicated significant differences between substrates $\left(p<0.001\right.$ ) on $\alpha$-diversity level (defined by $\mathrm{H}^{\prime}$; Figure 3A) and in general community composition represented by $\beta$-diversity $(p<0.05)$, respectively. Significant pairwise differences $(p<0.001)$ were further confirmed via pairwise t-test and Tukey's HSD post hoc test (Supplementary Table 3). Firmicutes were found to be the most dominant phyla in both FWD and OWD with $92 \%$ and $57 \%$ relative abundance. CFD was dominated by Proteobacteria (44\%) and contained overall lower abundances of Firmicutes (23\%). Bacteroidota were most common in OWD (38\%) but less abundant in FWD and CFD (28\% and $14 \%$, respectively).

With a share of $34.7 \pm 3.5 \%$, sparse OTUs with a relative abundance smaller than $1 \%$ contributed to a large part to the community in CFD compared with $8.9 \pm 0.4 \%$ in OWD and $5.2 \pm 0.8 \%$ in FWD. $H^{\prime}$ further indicated a heterogeneous distribution of abundances among few OTUs in FWD $\left(H^{\prime}=1.14 \pm 0.14\right)$ described by a low evenness $(J=0.33 \pm 0.03)$. CFD was defined by a more even community $(J=0.79 \pm 0.03)$ consisting of many less abundant OTUs $\left(H^{\prime}=4.15 \pm 0.21\right)$. For matters of clarity, only genera with abundances of $>5 \%$ were included in Figure 3B. On genus level, FWD was largely dominated by Leuconostoc (77\%) of the phylum Firmicutes. In OWD $\left(H^{\prime}=2.67 \pm 0.02\right.$; $J=0.66 \pm 0.02$ ), Firmicutes were mainly represented by unclassified Selenomonadaceae and shared their predominance (31\%) with a similarly abundant Prevotella (36\%).

\section{Taxonomic Exploration of the Gut Microbiota}

Sequencing of the $16 \mathrm{~S}$ rRNA V4 genetic region yielded a total of $3.50 \times 10^{6}$ reads from 36 BSFL gut samples (three biological replicates per time $\times$ treatment) after passing through quality control and filtering steps. Mantel tests on distance matrices 


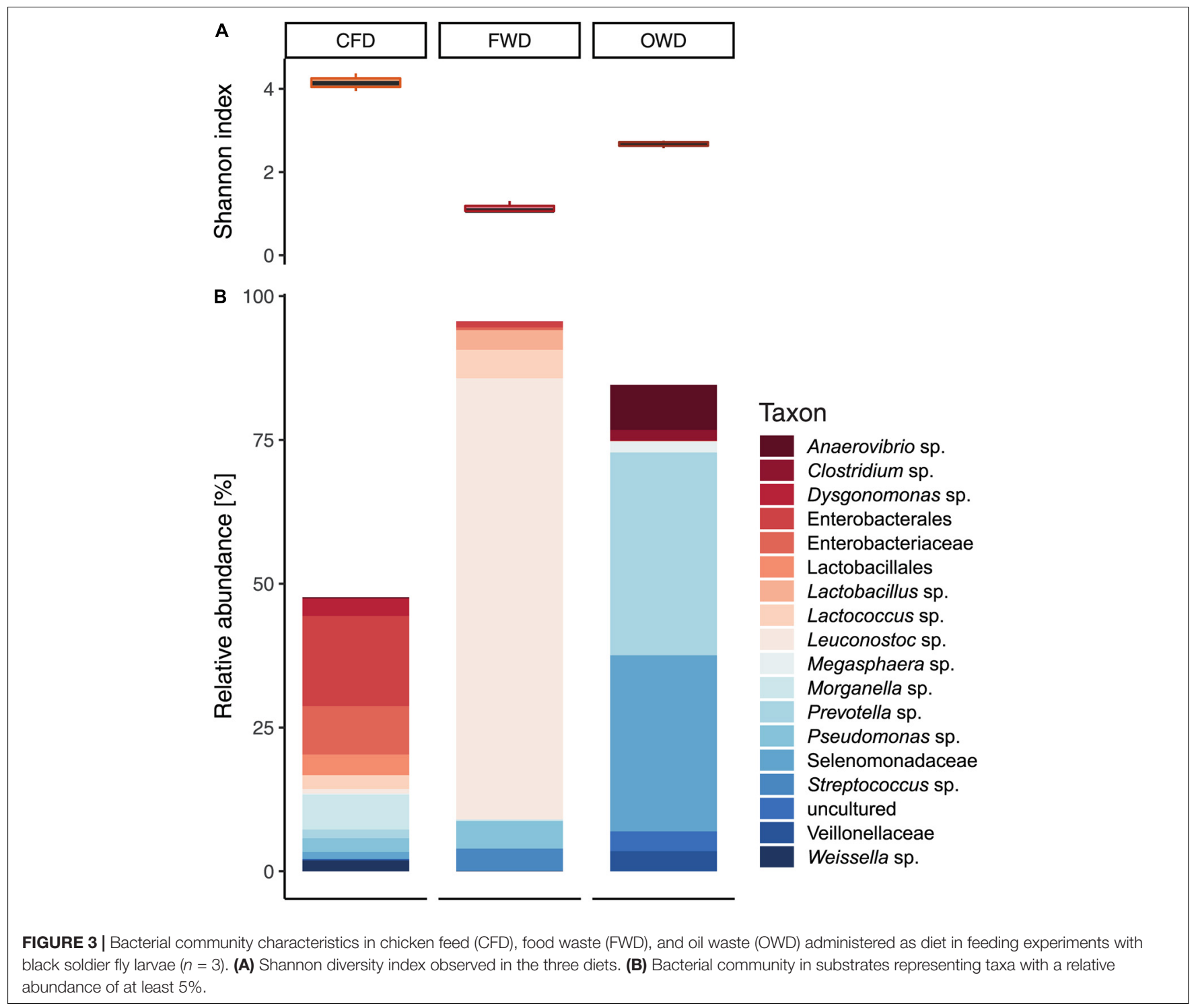

produced by the different clustering and subsampling approaches described in section "Data Analysis of Sequencing Data" resulted in no significant differences among the compared methods. This confirmed the overall congruency of the results irrespective of clustering method ( $97 \%$ similarity OTUs or unique ASVs) and subsampling size. The presented results are based on $97 \%$ similarity data subsampled to 36,878 reads (smallest sample size). The reads were clustered into 1,583 OTUs representing 491 genera within 39 phyla. Most abundant phyla across all guts were Firmicutes (30\%), Proteobacteria (26\%), Bacteroidota (15\%), and Actinobacteriota (7\%). The H' as well as J observed in the original population of 6 -day-old larvae (INI; $H^{\prime}=1.89 \pm 0.05$, $J=0.37 \pm 0.01)$ remained stable throughout the CFD treatment $\left(H^{\prime}=1.65 \pm 0.04, J=0.35 \pm 0.01\right)$ but significantly decreased in later samples obtained from the FWD treatment $\left.\left(H^{\prime}=0.59 \pm 0.18, J=0.13 \pm 0.04\right), p<0.05\right)$ (Figure 4A). A similar decrease was observed in later samples from OWD-fed larvae $\left(H^{\prime}=1.25 \pm 0.07, J=0.25 \pm 0.02\right)$.
PERMANOVA based on Bray-Curtis dissimilarity of gut microbiome data confirmed the observed differences in larval gut microbiomes $(p<0.001)$ between sampling time points and dietary treatments. Pairwise PERMANOVA with samples summarized on diet level specified significant compositional divergence from INI in the gut microbiome of FWD and OWD-fed larvae but showed stability in the CFD control group (Figure 4B and Supplementary Table 3). While Dysgonomonas (38\%) and Providencia (30\%) were initially highly prevalent in FWD, over time they were displaced by Morganella (from 1\% in $\mathrm{d}_{2}$ to $88 \%$ in $\mathrm{d}_{20}$; Figure $4 \mathrm{~B}$ ). A comparable development was observed in OWD, which contributed to an increasing similarity to FWD-fed larvae. NMDS analysis of the larval gut microbiome data (stress < 0.2) further explained spatial relationships between diets and highlighted the compositional overlap found in FWD and OWD-fed larvae (Figure 5A). Linear discriminant analysis of effect size found 30 OTUs to be explanatory for differences between groups, while 49 OTUs were identified to be strongly 
A

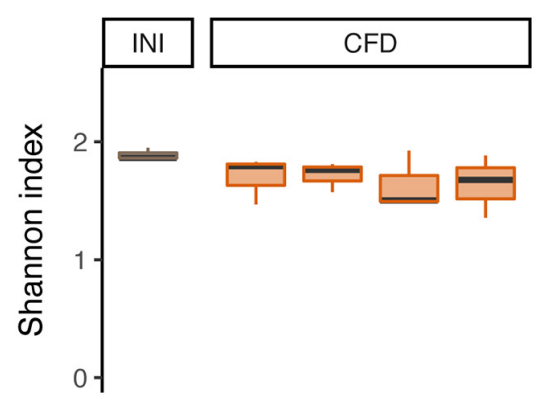

B

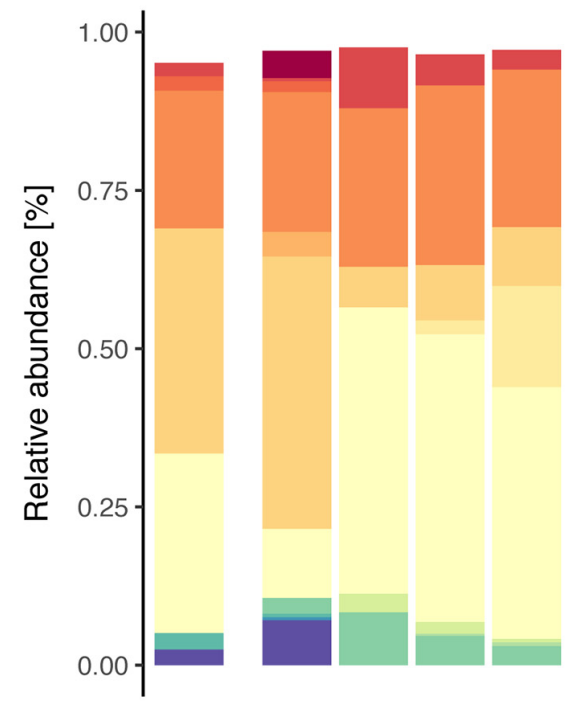

C

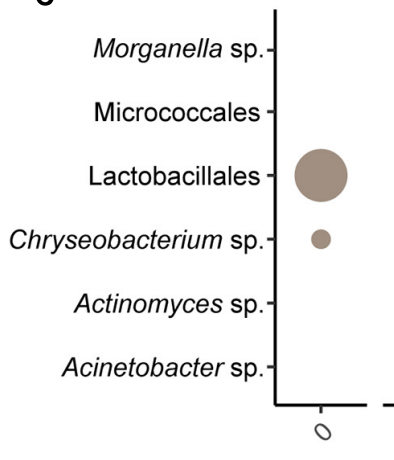

\author{
.
}
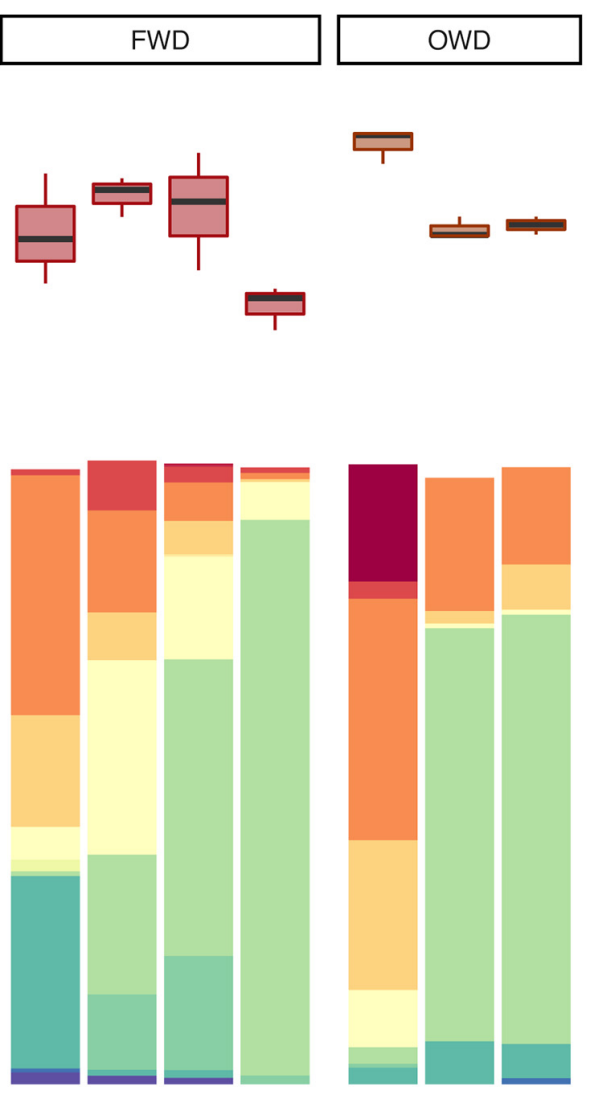

\section{Taxon}

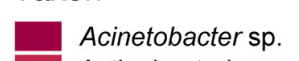

Actinobacteria

Actinomyces sp.

Chryseobacterium sp.

Dysgonomonas sp.

Empedobacter sp.

Enterobacteriaceae

Escherichia-Shigella sp.

Lactobacillales

Lactobacillus sp.

Micrococcales

Morganella sp.

Orbaceae

Providencia sp.

Sphingobacterium sp.

Vagococcus sp.

Weissella sp.

FIGURE 4 | Bacterial community characteristics in guts of black soldier fly larvae reared on chicken feed (CFD), food waste (FWD), and oil waste diet (OWD). Before introducing new diets, neonate larvae were reared on CFD for 6 days (INI). Data from each time point is presented as summary of three replicates $(n=3)$.

(A) Shannon diversity index observed in guts of BSFL. (B) Bacterial community composition in guts of BSFL representing taxa with a relative abundance of at least 1\%. (C) Indicator species identified by both linear discriminant analysis of effect size and multi-level pattern analysis with a relative abundance of at least $1 \%$.

associated with the respective dietary treatment by multi-level pattern analysis. A congruent set of 17 OTUs recognized by both methods was defined as indicator species and assigned to 15 distinct genera (genera with relative abundance $>1 \%$ in Figure 4C). Representatives of the order of Lactobacillales were found to be characteristic in guts of all treatments, but could not be assigned to a described genus. Morganella contributed with high abundances to the differentiation of mid to late stages of FWD- and OWD-fed larvae from the control group. However, CFD-fed larvae contained specific differentially abundant bacterial groups depending on their stage of growth. Chryseobacterium was found to be distinctive for early stages, while Actinomyces and Micrococcales were mostly found in mid and late stages. Moreover, the later samples spread further away from the initial community (INI) that consisted of predominantly Enterobacteriaceae (36\%), Lactobacillales (29\%), and Dysgonomonas (21\%). Although similar in larval development time and growth rate, CFD and FWD-fed larvae 

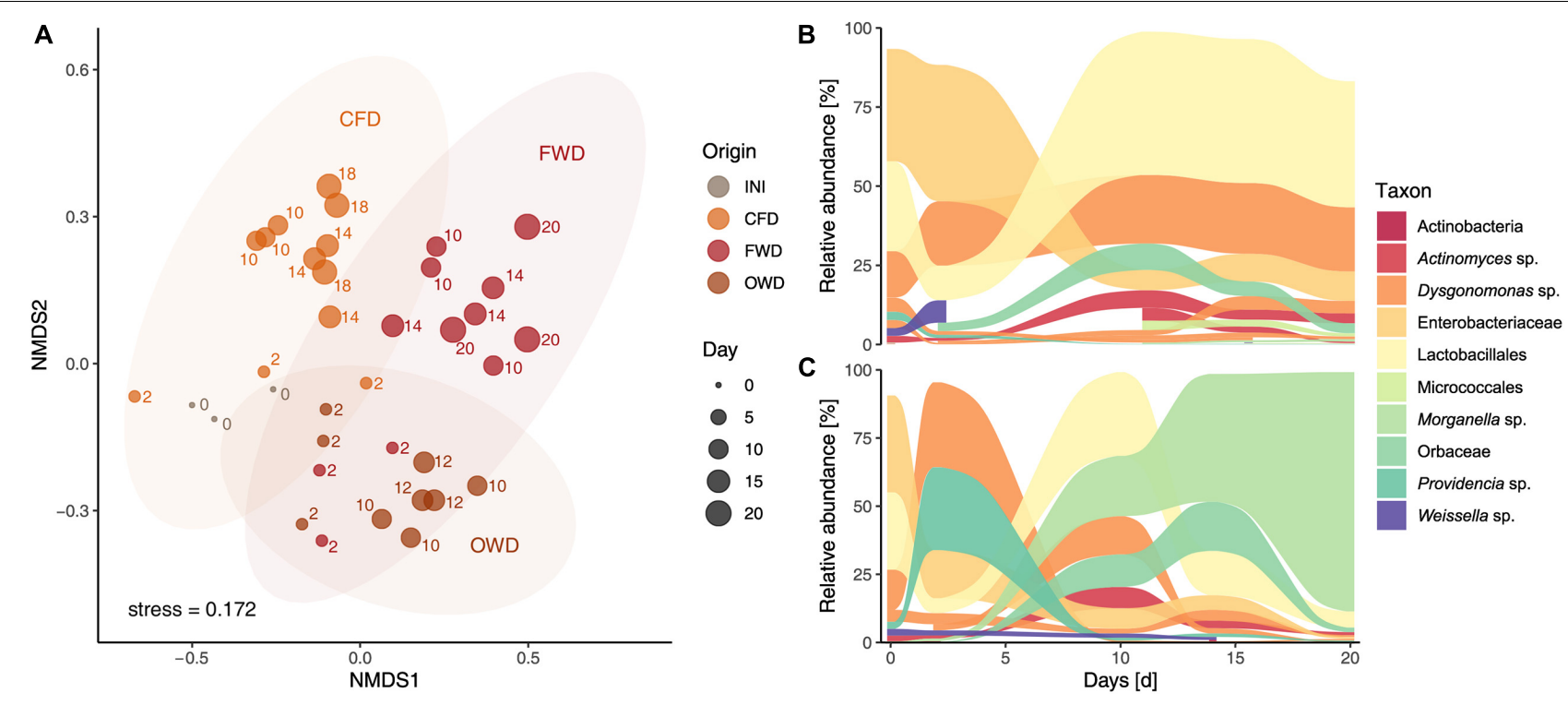

FIGURE 5 | Compositional relationships and dynamics in black soldier fly guts $(n=3)$. (A) Non-metric multidimensional scaling of larval gut microbiome data based on Bray-Curtis dissimilarity with ellipses representing a 95\% confidence interval. Data was filtered to contain OTUs with a prevalence of four in at least $10 \%$ of the samples. INI, initial population of 6-day old larvae; CFD, chicken feed diet; FWD, food waste diet; OWD, oil waste diet. Dynamics of taxa with a relative abundance $>1 \%$ present in at least three sampling events in black soldier fly larvae reared on chicken feed (B) and food waste (C).

exhibited different dynamics of the most abundant taxa in their guts. Persistent genera with a relative abundance $>1 \%$ and present in at least three sampling events are shown in Figures 5B,C for CFD and FWD-fed larvae, respectively.

\section{Imputation of Main Metabolic Pathways of the Gut Microbiota}

Approaches making use of metagenomic imputation as introduced by the $\mathrm{R}$ packages Tax4Fun2 were used to infer metabolic pathways from $16 \mathrm{~S}$ rRNA gene-based amplicon data. On average, $70 \pm 18 \%$ of the obtained sequences could be assigned to the reference database and used for the prediction of pathways. PERMANOVA analysis indicated significant timeand diet-dependent differences $(p<0.001)$ in metabolic profiles (Supplementary Table 3). Pairwise comparison of data grouped on diet-level via pairwise PERMANOVA revealed that both OWD and FWD differ from the CFD control group, but only OWD diverged from the metabolic pathways found in larval gut communities of INI. In contrast to the deviating development of FWD from INI on a phylogenetic level, this difference was not observed in a metabolic context. In general, the distinction between treatments on the level of metabolic pathways was much blurrier than on a taxonomic level (Figures 4B, 6).

\section{DISCUSSION}

Two major fractions of canteen waste - FWD and OWD - were compared with a commonly used CFD. Impact on the growth, the gut microbiome profiles, and the divergence from the initial 6-day old population of BSFL were determined.

\section{Canteen Food Waste as an Easily Degradable and Growth-Promoting Substrate for BSFL}

Food waste from canteens is highly heterogenous and mainly consist of staples (potatoes, rice, pasta), vegetables/fruits, and grain products with smaller amounts of meat and fish wastes (Silvennoinen et al., 2015). Our FWD derived from one batch of mixed organic wastes that accumulated on a single day in a local canteen. We found that larvae fed FWD took a highly similar course in biomass gain and development as the CFDfed control group (Figures 2A,B). However, endpoint biomass of FWD-fed larvae were $5 \%$ below the average measured in the control. These results are congruent with previously published records of larvae raised on chicken feed (on average, $158 \pm 20 \mathrm{mg}$ larva $^{-1}$; Barragán-Fonseca et al., 2017). While CFD-fed larvae did not experience a considerable decrease in biomass once they had reached their peak weight, FWD-fed larvae approaching (pre-)pupal stage consistently lost mass. This is crucial in calculating cost efficiency and designing processes in the largescale application of BSFL (Salomone et al., 2017). A biomass peak significantly higher a few days prior to the transition to pupal stage suggests not to use the self-harvesting ability of larvae in an industrial setting. Instead, a batch system with scheduled mechanical or manual harvesting cycles taking place during the fifth instar before onset of migration could represent a more efficient option. Although greenhouse gas emission and overall environmental footprint of treating organic wastes with BSFL were already low compared with traditional composting (Mertenat et al., 2019), timing the harvest of larvae could boost the efficiency of operating resources. Improved biomass output and more stable frequency of rearing cycles would be the 


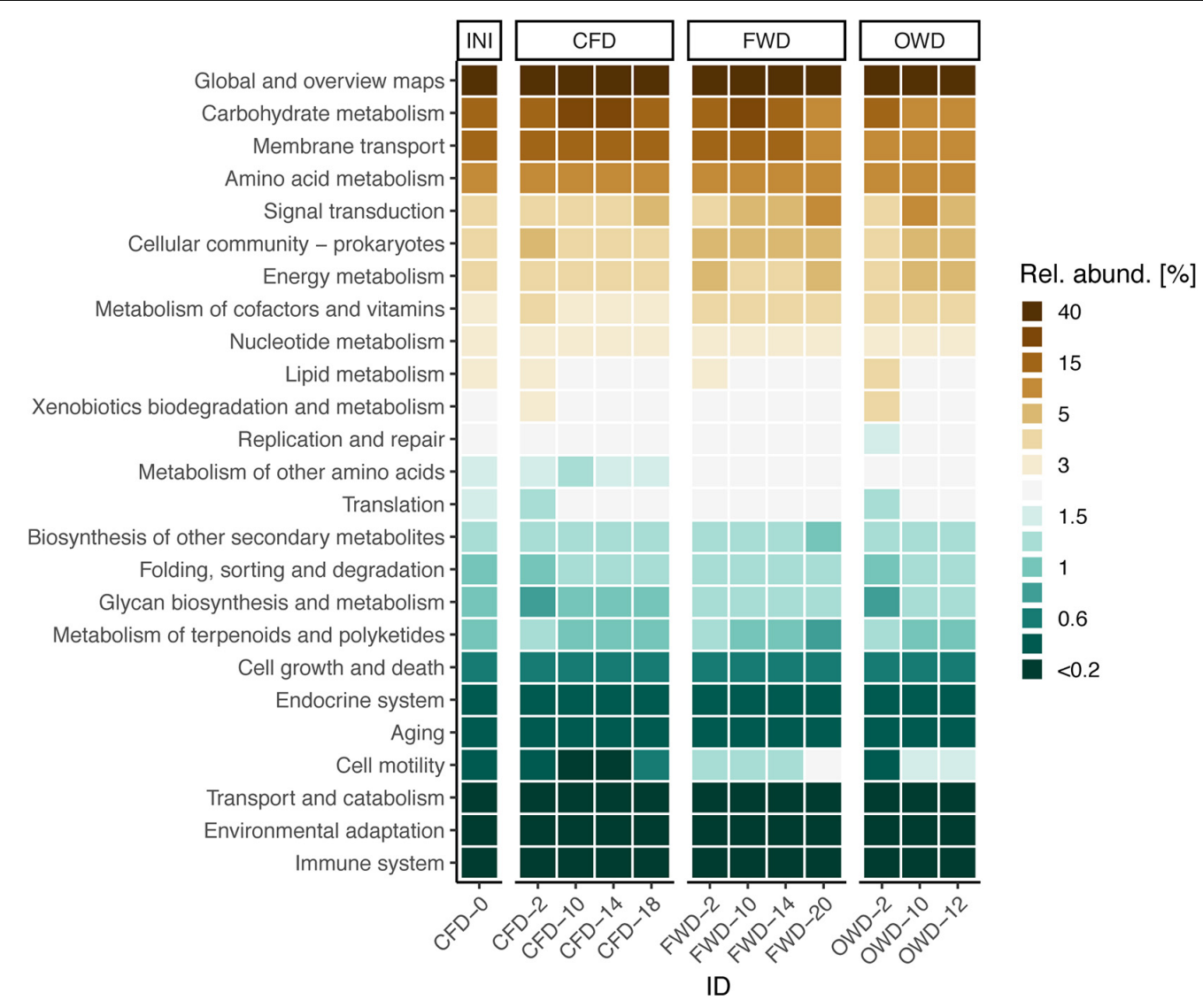

FIGURE 6 | Most abundant functional pathways in the gut microbiota of black soldier fly larvae before introduction of dietary treatments (INI) and after introduction of food waste (FWD) and oil waste (OWD) $(n=3)$. The control group was further fed with chicken feed (CFD). Pathways were obtained from Tax4Fun2 analysis and summarized on level 2 of the BRITE hierarchy based on KEGG orthology.

consequences. The advantages of harvest before the last instar became also evident when larvae were used for the production of biodiesel, since they contained a higher fraction of lipids and less chitin (Wong et al., 2019).

During the degradation process, FWD was highly modified by larval and microbial activity. This is indicated by high concentrations of VFAs such as valerate and butyrate, but also higher $\mathrm{NH}_{4}$ contents (Figure 2C). The accumulation of nitrogen compounds is of interest for the commercialization of residues as soil amendment (Klammsteiner et al., 2020a). Residues from BSFL-processed household organic wastes showed similar $\mathrm{C}_{\text {tot }}$ and $\mathrm{N}_{\text {tot }}$ ratios to the FWD-R produced in our study and have been identified as an effective nitrogen source for plant growth (Kawasaki et al., 2020). Moreover, the generally high chitin content in residues produced by insects can improve the defense against phytopathogens by triggering plant-based immune responses (Sharp, 2013).

When food waste remains untreated, microbial spoilage dominated by lactic acid bacteria can cause the $\mathrm{pH}$ to rapidly drop to approx. 4.3 within $7 \mathrm{~h}$ (Aichinger et al., 2015; Wu et al., 2018). A similarly low initial pH of 4.5 was observed in the food waste fed to the larvae in our study (Supplementary Table 1), before shifting to a more neutral value in post-process residues (Figure 2C). This is particularly important from a hygiene perspective, as these acidic conditions can inhibit bacterial pathogens (Wu et al., 2018). In addition, BSFL have been shown to exhibit antimicrobial activity against multiple potentially human pathogenic bacteria by excreting antimicrobial compounds into their environment (Erickson et al., 2004; Liu et al., 2008; Choi et al., 2012).

Larvae fed with FWD showed significantly enhanced substrate reduction, better approximate digestibility of the substrate, and a higher consumption index compared with CFD (Table 1). However, a presumably lower nutrient availability in this diet required the ingestion of larger amounts to reach the same larval biomass as was observed in CFD (Table 2). This is emphasized by the comparably low efficiency of FWD-fed larvae to convert ingested and digested food to their own biomass. The chicken feed commonly used to maintain BSF lab populations was developed as a balanced, energy-rich diet for laying hens and optimized amounts for BSFL rearing have been investigated by Diener et al. (2009). The overall benefit created by using waste-derived substrates, however, can make up for the lower biomass yield and slower development. It is still not known whether the larvae adapt to a specific diet over time and how many generations are necessary to establish metabolic 
stability. Nutrition-induced epigenetic inheritance from previous generations has been found to influence offspring phenotypes in other Dipterans such as Drosophila melanogaster (Wang et al., 2017). The potential distortion and its extent during impact assessment of newly introduced diets on BSFL growth and microbe-host relationships have yet to be quantified.

\section{Oil-Separator Waste Is Strongly Reducing BSFL Development and Survival}

The OWD treatment mainly derived from fats, oils, and grease (FOG) trapped as supernatant in the collection container of an oil separator. In addition to the lipid fraction, it also contained a smaller and heterogenous fraction of food residues from the cooking process. The fatty components of the OWD represent a more problematic fraction of the canteen waste since FOGs generally require more refined treatment methods (Husain et al., 2014). Supplying them to anaerobic digestion is a frequently chosen biotreatment (Long et al., 2012), but also composting of FOG compounds provided promising results (Lemus and Lau, 2002).

In our trial, the OWD changed its consistency within a few days and became more viscous, making it harder for the larvae to move. However, physicochemical properties and volatile fatty acid profiles remained largely unchanged (Figures 2C,D and Supplementary Tables 1, 2). Solidification and deposition of FOGs are a widespread problem in sewer systems transporting wastewater from food service establishments (He et al., 2013). These processes are most likely driven by high concentrations of saturated fats and calcium, while moisture content is secondary (Keener et al., 2009). Due to the slow growth rate and negligible substrate reduction, the degradation parameters did not provide any meaningful information.

A combination of inhibited mobility and lack of easily degradable nutrients may have led to the starvation of the larvae. Moreover, the physical blocking of the larvae's spiracles by the oil could have led to their suffocation (Taverner, 2002). After approximately 7 days, the extent of this effect became visible by an exponentially increasing mortality (Figure 2A). No notable ingestion and digestion of the substrate could be assessed by the consumption and waste reduction index (Table 1). There have not been in-depth investigations on BSF lipid metabolism yet, but a recent study showed that BSFL do not only bioaccumulate fatty acids from their diet but are also able to synthesize fatty acids themselves (Hoc et al., 2020). Future studies with diets supplemented with defined combinations of FOGs could determine limits of BSFL fat tolerance in organic wastes to enable FOG utilization in BSFL feedstuff.

\section{Food Waste Diet Shapes the BSFL Gut Microbiome}

Chicken feed diet-fed larvae retained a stable bacterial diversity throughout the experiment and did not diverge microbiomewise from the initial population of 6-day old larvae raised on the same diet (Figures 4A,B). These observations are in line with Cifuentes et al. (2020), who found that a chicken-feed-based diet contributes to an overall stable gut microbiome during larval development. The introduction of FWD and OWD treatments had a decisive impact on the development of the BSFL gut microbiome. Over time, the initially abundant Lactobacillales, Enterobacteriaceae, and Dysgonomonas were displaced by mostly Morganella in both diets. This compositional change further led to a decrease in $\alpha$-diversity by crowding-out other groups and thereby. Even though larval growth (Table 2) and substrate degradation parameters (Table 1) reported negligible conversion of OWD, the gut microbiomes of these larvae developed similarly to FWD-fed larvae (Figure 4B). However, in contrast to Raimondi et al. (2020), we could not observe a meaningful increase of gut microbiome complexity over time.

As recently stated by Raimondi et al. (2020), Proteobacteria, especially Providencia, dominated guts of vegetable-fed larvae irrespective of the rearing temperature used in their study. In our trial, however, Providencia was mostly found in early larval stages and in low abundances throughout the development of OWDfed larvae. This discrepancy is likely caused by the nutritional differences between their standard vegetable diet and the FWD and OWD used in our study. Larval ingestion of corn flour, wheat bran, and alfalfa flour contained in the vegetable diet might favor the prevalence of Providencia (Raimondi et al., 2020).

Furthermore, the Gram-negative and motile genus of Morganella, predominant in FWD and OWD-fed larvae, is known to share several biochemical aptitudes such as the (oxidative) deamination of certain amino acids with Providencia (Manos and Belas, 2006). It has previously been found to play a significant role in the BSFL gut microbiome due to its prevalence across various feeding schemes and larval developmental stages (Wynants et al., 2018; Cifuentes et al., 2020; Klammsteiner et al., 2020b; Liu et al., 2020; Raimondi et al., 2020). In our study, Morganella strongly contributed as indicator species to the distinction of FWD- and OWD-shaped gut microbiomes from the control group (Figure 4C). It is an environmentally widespread genus of the Enterobacterales so far only consisting of the species Morganella morganii and is often found as commensal in human guts (Manos and Belas, 2006). Due to its potential to cause severe infections, it has been cited as a relevant pathogenic microorganism associated with insects grown for feed and food (Schlüter et al., 2017; Raimondi et al., 2020). As opposed to rearing BSFL raised on CFD, food waste favored the enrichment of Morganella in larval guts. In fresh FWD and OWD substrates, Morganella was only present in comparatively low relative abundances of $0.1-0.3 \%$ while fresh CFD contained $6 \%$ but did not promote its accumulation in larvae. Additionally, extensive gut colonization by other bacteria was inhibited as has previously been observed in carrion degrading burying beetles: while endogenous $M$. morganii not only contributed to the stabilization of the beetle gut microbiome, it also helped to outcompete rival bacterial communities and prevent colonization by potentially entomopathogenic bacteria (Duarte et al., 2018; Wang and Rozen, 2018). High abundances of this species were also reported for other Diptera such as wild populations of the Mediterranean fruit fly Ceratitis capitata (De Cock et al., 2019). On the contrary, for mass-reared Mexican fruit flies (Anastrepha ludens), inoculating the substrate with only $105 \mathrm{CFUs} \mathrm{ml}^{-1}$ 
of M. morganii led to $100 \%$ mortality in larvae while lower concentrations reduced emergence and flight ability of adults (Salas et al., 2017). Although Morganella did not negatively alter BSFL performance in our trial, the role of other entomopathogens as a risk factor in industrial $H$. illucens production remains to be investigated. Immunological responses of larvae in form of antimicrobial peptides have been addressed (Vogel et al., 2018). However, microorganisms (bacteria, fungi, and viruses) potentially endangering the uprising BSF industry - this includes fly populations as well as workplace safety - are due to be characterized (Joosten et al., 2020).

Since previously reported studies rarely match in rearing conditions, protocols, and locations, detailed comparisons are strongly limited (De Smet et al., 2018). Shifts, and thereby adaptations, of gut microbial communities to new diets might be primarily driven by the functional needs involved in degrading the available substrate. By clarifying whether in BSFL metabolic competence is preferred over enrichment of ingested exogenous microbes, the accumulation dynamics of specific phylogenetic groups in the larval gut could further be explained. In this context, investigating also the modification of the inherent digestive enzymatic toolkit and gut cell morphology in relation to diet uptake constitutes a crucial approach to gain a comprehensive picture of larval metabolism (Bruno et al., 2019; Bonelli et al., 2020). Nevertheless, we think that especially the pursuit to convert organic wastes known to carry a high bioburden into larval biomass should provide further motivation to find appropriate pre-treatment methods for substrates.

\section{Indicator Species as Traits for Dietary Adaptation of Microbial Communities in the Gut}

Besides Morganella, Lactobacillales pervaded guts from all treatments (Figure 4C). They have been identified to be a common driving factor for the divergence of dietary treatment groups. Although the OTUs representative of this group could not be classified at the genus level, a selection of Lactobacillales taking place based on the ingested diet is probable. This order also includes lactic acid bacteria such as Lactobacillus, Leuconostoc, and Lactococcus. In extensively studied honeybees and fruit flies, probiotic lactic acid bacteria were found to improve pesticide resistance and gastrointestinal pathogen control (Trinder et al., 2015; Daisley et al., 2017). Moreover, inoculation of organic wastes with Lactobacilli was shown to yield a higher biomass output and a better nutritional spectrum in BSFL compared with artificial feed-amended wastes (Somroo et al., 2019). Depending on their distribution at a higher phylogenetic resolution (e.g., species level), Lactobacillales identified as indicator species could act as candidate biomarker for larva-substrate interactions. Wu et al. (2018) found that lactic acid bacteria, and in particular Lactobacillales, strongly prevail during the microbial degradation of food waste and shape its microbiome by changing physicochemical parameters.

In the CFD-fed control group, a temporal succession of low-abundant indicator species was observed. Chryseobacterium defined the guts of early CFD-fed larvae and was previously linked to the digestion of high-fiber diets in guts of American cockroaches (Dugas et al., 2001). Two Actinobacteria represented by Actinomyces and Micrococcales, were found to be specific for mid to late-stage larvae. These findings are consistent with observations made by Raimondi et al. (2020), who identified Micrococcales (in particular Brevibacterium) as biomarker in prepupae. In a previous study, we identified Actinomyces as a main member of the gut core microbiome in larvae raised on low-bioburden diets such as commercial chicken feed (Klammsteiner et al., 2020b).

\section{Main Metabolic Features Are Not Primarily Defined by Community Composition}

Deducing metabolic pathways from short reads and microbial abundances comes with limitations but can maximize the informative value of marker-gene data. Especially environmental samples have more shortcomings compared with human-derived samples. Poorer availability of closely related reference genomes and the reliance on comparatively short sequence fragments that are often highly similar among distinct bacterial families have to be kept in mind (Knight et al., 2018). However, deducing links between taxonomy and function from $16 \mathrm{~S}$ rRNA gene-based amplicon data can also be a preferable method to shotgun metagenomic sequencing, when, e.g., the host genome DNA strongly interferes with the microbiome signal at sequencing step (Langille, 2018). This could pose a problem when using metagenomic sequencing to investigate gut microbial communities in BSFL, as samples still contain host derived tissues.

Because of the known limitations, we decided to conduct our comparative analysis on the second level of the KEGG hierarchy instead of focusing on distinct pathways and/or metabolic functions. Although the administered diets induced clear shifts in the composition of microbial communities, the differences in the main metabolic pathways between dietary treatments were not as clear (Figure 6). A similar conclusion was reached by Zhan et al. (2019) on the larval transcriptome level: to help with the digestion of food waste as well as poultry, swine, and dairy manure, larvae use a common genetic toolkit instead of expressing diet-specific genes. These basic findings are directly in line with findings of The Human Microbiome Project Consortium (2012). A comprehensive study of the human microbiome led to the conclusion that metabolic pathways are mostly stable despite larger variations in microbial community structure. Pathways taking care of general and specific metabolic functions (carbohydrate, amino acid, energy, cofactor, and vitamin metabolism), membrane transport, and cell communication were amongst the highest abundant pathways in BSFL gut microbiomes.

So far, the investigation of metabolic functions of the BSFL gut microbiome has received little attention. Integrating metagenomic and metatranscriptomic analyses in future studies as expansion of imputed metagenomics could help to find a consensus in describing the functional framework in which BSFL convert organic wastes to biomass. 


\section{CONCLUSION}

Decentralized collection and processing centers in hubs of social activity could represent a feasible way to valorize food waste. By premixing the wastes collected from various sources, a more average and stable larval feedstuff composition could be achieved. The high content of fat, oil, and grease in the oil waste fraction of canteen wastes was found to be lethal for BSFL, but the conversion of the food waste fraction proceeded efficiently. Dietinduced shifts in gut microbiota were observed throughout the larval development. In contrast to the diet adaption on a phylogenetic level, general functional competences described as metabolic pathways resulted in similar patterns irrespective of the diet. This indicates that metabolic competence is a strong filter in the selection of larval gut colonizers, meaning that different communities can do the same job. Yet, many aspects of BSFL metabolism, especially fatty-acid digestion and accumulation as well as substrate-larva-microbiome interdependencies are still largely undescribed. Special attention should also be given to the assessment of risk factors such as entomopathogens and enrichment of potentially human-pathogenic microorganisms introduced by contaminated wastes.

\section{DATA AVAILABILITY STATEMENT}

The datasets presented in this study can be found in online repositories. The names of the repository/repositories and accession number(s) can be found below: https://www.ebi.ac.uk/ ena, PRJEB39545.

\section{AUTHOR CONTRIBUTIONS}

TK performed statistical and bioinformatical data analysis, created the figures, and wrote the manuscript. AW designed and supervised the experiment. TB carried out sample collection and preparation, physicochemical analyses, and extraction of

\section{REFERENCES}

Aichinger, P., Kuprian, M., Probst, M., Insam, H., and Ebner, C. (2015). Demanddriven energy supply from stored biowaste for biomethanisation. Bioresour. Technol. 194, 389-393. doi: 10.1016/j.biortech.2015.06.147

Aßhauer, K. P., Wemheuer, B., Daniel, R., and Meinicke, P. (2015). Tax4Fun: predicting functional profiles from metagenomic 16S rRNA data: Fig. 1. Bioinformatics 31, 2882-2884. doi: 10.1093/bioinformatics/btv287

Banks, I. J., Gibson, W. T., and Cameron, M. M. (2014). Growth rates of black soldier fly larvae fed on fresh human faeces and their implication for improving sanitation. Trop. Med. Int. Health 19, 14-22. doi: 10.1111/tmi.12228

Barragán-Fonseca, K. B., Dicke, M., and van Loon, J. J. A. (2017). Nutritional value of the black soldier fly (Hermetia illucens L.) and its suitability as animal feed a review. J. Insect Food Feed 3, 105-120. doi: 10.3920/JIFF2016.0055

Bonelli, M., Bruno, D., Brilli, M., Gianfranceschi, N., Tian, L., Tettamanti, G., et al. (2020). Black soldier fly larvae adapt to different food substrates through morphological and functional responses of the midgut. Int. J. Mol. Sci. 21:4955. doi: 10.3390/ijms21144955

Borrelli, L., Coretti, L., Dipineto, L., Bovera, F., Menna, F., Chiariotti, L., et al. (2017). Insect-based diet, a promising nutritional source, modulates
DNA. CH maintained the BSF colony, provided larvae for the experiment, and assisted during the experimental analyses. BS assisted with bioinformatical analysis and writing. FS, BS-S, and $\mathrm{HI}$ contributed equally to the study by supervising the planning, execution, and analysis of the study and provided scientific input during writing. All authors contributed important intellectual content and approved the final version of manuscript for publication.

\section{FUNDING}

This project was funded by the Austrian Science Fund (FWF, project number: P26444) and the Austrian Federal Ministry of Education, Science and Research (Marietta Blau Grant, ZI.: ICM2019-13390).

\section{ACKNOWLEDGMENTS}

We thank Jeff K. Tomberlin for providing valuable advice to establish our black soldier fly population, Sabina Vrečko for her work in the laboratory, and Andreas Wagner and Ljubica Begovic for their assistance in analytics. Thanks go to the Biotechnical Faculty and the Faculty of Civil Engineering of the University of Ljubljana for hosting TK during the bioinformatical analysis and manuscript writing. Moreover, we are grateful for the advice from the Unit of Environmental Engineering (IUT, University of Innsbruck) for the sampling of oil wastes. The computational results presented here have been achieved in part using the LEO HPC infrastructure of the University of Innsbruck.

\section{SUPPLEMENTARY MATERIAL}

The Supplementary Material for this article can be found online at: https://www.frontiersin.org/articles/10.3389/fmicb. 2021.619112/full\#supplementary-material

gut microbiota composition and SCFAs production in laying hens. Sci. Rep. 7:16269. doi: 10.1038/s41598-017-16560-6

Bosch, G., Oonincx, D. G. A. B., Jordan, H. R., Zhang, J., van Loon, J. J. A., van Huis, A., et al. (2020). Standardisation of quantitative resource conversion studies with black soldier fly larvae. J. Insect Food Feed 6, 95-109. doi: 10.3920/ JIFF2019.0004

Boschini, M., Falasconi, L., Cicatiello, C., and Franco, S. (2020). Why the waste? A large-scale study on the causes of food waste at school canteens. J. Clean Prod. 246:118994. doi: 10.1016/j.jclepro.2019.118994

Breitwieser, F., Lu, J., and Salzberg, S. (2017). A review of methods and databases for metagenomic classification and assembly. Brief Bioinform. 20, 1125-1136. doi: 10.1093/bib/bbx120

Bruno, D., Bonelli, M., Cadamuro, A. G., Reguzzoni, M., Grimaldi, A., Casartelli, M., et al. (2019). The digestive system of the adult Hermetia illucens (Diptera: Stratiomyidae): morphological features and functional properties. Cell Tissue Res. 378, 221-238. doi: 10.1007/s00441-019-03025-7

Caporaso, J. G., Lauber, C. L., Walters, W. A., Berg-Lyons, D., Lozupone, C. A., Turnbaugh, P. J., et al. (2011). Global patterns of 16S rRNA diversity at a depth of millions of sequences per sample. PNAS 108, 4516-4522. doi: 10.1073/pnas. 1000080107 
Chen, B., Teh, B.-S., Sun, C., Hu, S., Lu, X., Boland, W., et al. (2016). Biodiversity and activity of the gut microbiota across the life history of the insect herbivore Spodoptera littoralis. Sci. Rep. 6:29505. doi: 10.1038/srep29505

Cho, S., Kim, C.-H., Kim, M.-J., and Chung, H. (2020). Effects of microplastics and salinity on food waste processing by black soldier fly (Hermetia illucens) larvae. J. Ecol. Environ. 44:7. doi: 10.1186/s41610-020-0148-x

Choi, W.-H., Yun, J.-H., Chu, J.-P., and Chu, K.-B. (2012). Antibacterial effect of extracts of Hermetia illucens (Diptera: Stratiomyidae) larvae against Gramnegative bacteria. Entomol. Res. 42, 219-226. doi: 10.1111/j.1748-5967.2012. 00465.x

Cifuentes, Y., Glaeser, S. P., Mvie, J., Bartz, J.-O., Müller, A., Gutzeit, H. O., et al. (2020). The gut and feed residue microbiota changing during the rearing of Hermetia illucens larvae. Antonie van Leeuwenhoek 113, 1323-1344. doi: 10. 1007/s10482-020-01443-0

Daisley, B. A., Trinder, M., McDowell, T. W., Welle, H., Dube, J. S., Ali, S. N., et al. (2017). Neonicotinoid-induced pathogen susceptibility is mitigated by Lactobacillus plantarum immune stimulation in a Drosophila melanogaster model. Sci. Rep. 7:2703. doi: 10.1038/s41598-017-02806-w

De Cáceres, M., and Legendre, P. (2009). Associations between species and groups of sites: indices and statistical inference. Ecology 90, 3566-3574. doi: 10.1890/ 08-1823.1

De Cock, M., Virgilio, M., Vandamme, P., Augustinos, A., Bourtzis, K., Willems, A., et al. (2019). Impact of sample preservation and manipulation on insect gut microbiome profiling: a test case with fruit flies (Diptera, Tephritidae). Front. Microbiol. 10:2833. doi: 10.3389/fmicb.2019.02833

De Smet, J., Wynants, E., Cos, P., and Campenhout, L. V. (2018). Microbial community dynamics during rearing of black soldier fly larvae (Hermetia illucens) and its impact on exploitation potential. Appl. Environ. Microbiol. 84, 2722-2717. doi: 10.1128/AEM.02722-17

Diener, S., Zurbrügg, C., and Tockner, K. (2009). Conversion of organic material by black soldier fly larvae: establishing optimal feeding rates. Waste Manag. Res. 27, 603-610. doi: 10.1177/0734242X09103838

Douglas, G. M., Maffei, V. J., Zaneveld, J. R., Yurgel, S. N., Brown, J. R., Taylor, C. M., et al. (2020). PICRUSt2 for prediction of metagenome functions. Nat. Biotechnol. 38, 685-688. doi: 10.1038/s41587-020-0548-6

Duarte, A., Welch, M., Swannack, C., Wagner, J., and Kilner, R. M. (2018). Strategies for managing rival bacterial communities: Lessons from burying beetles. J. Anim. Ecol. 87, 414-427. doi: 10.1111/1365-2656.12725

Dugas, J. E., Zurek, L., Paster, B. J., Keddie, B. A., and Leadbetter, E. R. (2001). Isolation and characterization of a Chryseobacterium strain from the gut of the American cockroach, Periplaneta americana. Arch. Microbiol. 175, 259-262. doi: $10.1007 / \mathrm{s} 002030000243$

Erickson, M. C., Islam, M., Sheppard, C., Liao, J., and Doyle, M. P. (2004). Reduction of Escherichia coli o157:h7 and Salmonella enterica serovar enteritidis in chicken manure by larvae of the black soldier fly. J. Food Prot. 67, 685-690. doi: 10.4315/0362-028x-67.4.685

FAO (2011). Global food losses and food waste: extent, causes and prevention. Rome: Food and Agriculture Organization of the United Nations.

He, X., de los Reyes, F. L., Leming, M. L., Dean, L. O., Lappi, S. E., and Ducoste, J. J. (2013). Mechanisms of fat, oil and grease (FOG) deposit formation in sewer lines. Water Res. 47, 4451-4459. doi: 10.1016/j.watres.2013.05.002

Heussler, C. D., Walter, A., Oberkofler, H., Insam, H., Arthofer, W., Schlick-Steiner, B. C., et al. (2018). Influence of three artificial light sources on oviposition and half-life of the black soldier fly, Hermetia illucens (Diptera: Stratiomyidae): Improving small-scale indoor rearing. PLoS One 13:e0197896. doi: 10.1371/ journal.pone.0197896

Hoc, B., Genva, M., Fauconnier, M.-L., Lognay, G., Francis, F., and Caparros Megido, R. (2020). About lipid metabolism in Hermetia illucens (L. 1758): on the origin of fatty acids in prepupae. Sci. Rep. 10:11916. doi: 10.1038/s41598020-68784-8

Human Microbiome Project Consortium (2012). Structure, function and diversity of the healthy human microbiome. Nature 486, 207-214. doi: 10.1038/ nature 11234

Husain, I. A. F., Alkhatib, M. F., Jammi, M. S., Mirghani, M. E. S., Zainudin, Z. B., and Hoda, A. (2014). Problems, control, and treatment of fat, oil, and grease (FOG): a review. J. Oleo Sci. 63, 747-752. doi: 10.5650/jos.ess13182

Joosten, L., Lecocq, A., Jensen, A. B., Haenen, O., Schmitt, E., and Eilenberg, J. (2020). Review of insect pathogen risks for the black soldier fly (Hermetia illucens) and guidelines for reliable production. Entomol. Exp. Appl. 168, 432447. doi: 10.1111/eea.12916

Jucker, C., Lupi, D., Moore, C. D., Leonardi, M. G., and Savoldelli, S. (2020). Nutrient recapture from insect farm waste: bioconversion with Hermetia illucens (L.) (Diptera: Stratiomyidae). Sustainability 12:362. doi: 10.3390/ su12010362

Kanehisa, M., and Goto, S. (2000). KEGG: Kyoto Encyclopedia of Genes and Genomes. Nucl. Acids Res. 28, 27-30. doi: 10.1093/nar/28.1.27

Kassambara, A., and Mundt, F. (2020). factoextra: Extract and Visualize the Results of Multivariate Data Analyses. Vienna: R Core Team.

Kawasaki, K., Kawasaki, T., Hirayasu, H., Matsumoto, Y., and Fujitani, Y. (2020). Evaluation of fertilizer value of residues obtained after processing household organic waste with black soldier fly larvae (Hermetia illucens). Sustainability 12:4920. doi: $10.3390 / \mathrm{su} 12124920$

Keener, K., Ducoste, J., and Holt, L. (2009). Properties influencing fat, oil, and grease deposit formation. Water Environ. Res. 80, 2241-2246. doi: 10.2175/ 193864708 X267441

Klammsteiner, T., Turan, V., Juárez, M. F.-D., Oberegger, S., and Insam, H. (2020a). Suitability of black soldier fly frass as soil amendment and implication for organic waste hygienization. Agronomy 10:1578. doi: 10.3390/ agronomy10101578

Klammsteiner, T., Walter, A., Bogataj, T., Heussler, C. D., Stres, B., Steiner, F. M., et al. (2020b). The core gut microbiome of black soldier fly (Hermetia illucens) larvae raised on low-bioburden diets. Front. Microbiol. 11:993. doi: 10.3389/ fmicb.2020.00993

Klammsteiner, T., Walter, A., Pan, H., Gassner, M., Heussler, C. D., Schermer, M., et al. (2020c). "On everyone's lips: insects for food and feed," in Proceedings of 5th Austrian Citizen Science Conference 2019 - PoS(ACSC2019) (Trieste: SISSA Medialab), doi: 10.22323/1.366.0006

Knight, R., Vrbanac, A., Taylor, B. C., Aksenov, A., Callewaert, C., Debelius, J., et al. (2018). Best practices for analysing microbiomes. Nat. Rev. Microbiol. 16, 410-422. doi: 10.1038/s41579-018-0029-9

Kostanjšek, R., Prodan, Y., Stres, B., and Trontelj, P. (2019). Composition of the cutaneous bacterial community of a cave amphibian, Proteus anguinus. FEMS Microbiol. Ecol. 95:fiz007. doi: 10.1093/femsec/fiz007

Langille, M. G. I. (2018). Exploring linkages between taxonomic and functional profiles of the human microbiome. mSystems 3, 163-117e. doi: 10.1128/ mSystems.00163-17

Langille, M. G. I., Zaneveld, J., Caporaso, J. G., McDonald, D., Knights, D., Reyes, J. A., et al. (2013). Predictive functional profiling of microbial communities using 16S rRNA marker gene sequences. Nat. Biotechnol. 31, 814-821. doi: $10.1038 /$ nbt.2676

Lee, C.-M., Kim, S.-Y., Song, J., Lee, Y.-S., Sim, J.-S., and Hahn, B.-S. (2018). Isolation and characterization of a halotolerant and protease-resistant $\alpha$ galactosidase from the gut metagenome of Hermetia illucens. J. Biotechnol. 279, 47-54. doi: 10.1016/j.jbiotec.2018.05.003

Lemus, G. R., and Lau, A. K. (2002). Biodegradation of lipidic compounds in synthetic food wastes during composting. Can. Biosyst. Eng. 44, 33-36.

Liu, C., Yao, H., Chapman, S. J., Su, J., and Wang, C. (2020). Changes in gut bacterial communities and the incidence of antibiotic resistance genes during degradation of antibiotics by black soldier fly larvae. Environ. Int. 142:105834. doi: 10.1016/j.envint.2020.105834

Liu, Q., Tomberlin, J. K., Brady, J. A., Sanford, M. R., and Yu, Z. (2008). Black soldier fly (Diptera: Stratiomyidae) larvae reduce Escherichia coli in dairy manure. Environ. Entomol. 37, 1525-1530. doi: 10.1603/0046-225x-37.6.1525

Long, J. H., Aziz, T. N., de los Reyes, F. L., and Ducoste, J. J. (2012). Anaerobic codigestion of fat, oil, and grease (FOG): A review of gas production and process limitations. Process Saf. Environ. 90, 231-245. doi: 10.1016/j.psep.2011.10.001

Manos, J., and Belas, R. (2006). "The genera Proteus, Providencia, and Morganella," in The Prokaryotes, eds M. Dworkin, S. Falkow, E. Rosenberg, K.-H. Schleifer, and E. Stackebrandt (New York: Springer), 245-269. doi: 10.1007/0-38730746-X_12

Medrano, J. F., and Gall, G. A. E. (1976). Food consumption, feed efficiency, metabolic rate and utilization of glucose in lines of Tribolium castaneum selected for 21-day pupa weight. Genetics 83, 393-407.

Mertenat, A., Diener, S., and Zurbrügg, C. (2019). Black soldier fly biowaste treatment - assessment of global warming potential. Waste Manag. 84, 173-181. doi: 10.1016/j.wasman.2018.11.040 
Nakamura, S., Ichiki, R. T., Shimoda, M., and Morioka, S. (2016). Small-scale rearing of the black soldier fly, Hermetia illucens (Diptera: Stratiomyidae), in the laboratory: low-cost and year-round rearing. Appl. Entomol. Zool. 51, 161-166. doi: 10.1007/s13355-015-0376-1

Nguyen, T. T. X., Tomberlin, J. K., and Vanlaerhoven, S. (2015). Ability of black soldier fly (Diptera: Stratiomyidae) larvae to recycle food waste. Environ. Entomol. 44, 406-410. doi: 10.1093/ee/nvv002

Noble, J. E., and Bailey, M. J. A. (2009). “Chapter 8: quantitation of protein," in Methods in Enzymology Guide to Protein Purification, 2nd Edn, eds R. R. Burgess and M. P. Deutscher (Cambridge: Academic Press), 73-95. doi: 10.1016/S00766879(09)63008-1

Oksanen, J., Blanchet, F. G., Friendly, M., Kindt, R., Legendre, P., McGlinn, D., et al. (2018). vegan: Community Ecology Package. Vienna: R Core Team.

Principato, L. (2018). Food Waste at Consumer Level: A Comprehensive Literature Review. New York, NY: Springer International Publishing, doi: 10.1007/978-3319-78887-6

Quast, C., Pruesse, E., Yilmaz, P., Gerken, J., Schweer, T., Yarza, P., et al. (2013). The SILVA ribosomal RNA gene database project: improved data processing and web-based tools. Nucl. Acids Res. 41, D590-D596. doi: 10.1093/nar/gks1219

$\mathrm{R}$ Core Team (2018). R: a language and environment for statistical computing. Vienna: R Foundation for Statistical Computing.

Raimondi, S., Spampinato, G., Macavei, L. I., Lugli, L., Candeliere, F., Rossi, M., et al. (2020). Effect of rearing temperature on growth and microbiota composition of Hermetia illucens. Microorganisms 8:902. doi: 10.3390/ microorganisms 8060902

Rasmussen, L. B., Lassen, A. D., Hansen, K., Knuthsen, P., Saxholt, E., and Fagt, S. (2010). Salt content in canteen and fast food meals in Denmark. Food Nutr. Res. 54:2100. doi: 10.3402/fnr.v54i0.2100

Salas, B., Conway, H. E., Schuenzel, E. L., Hopperstad, K., Vitek, C., and Vacek, D. C. (2017). Morganella morganii (Enterobacteriales: Enterobacteriaceae) is a lethal pathogen of mexican fruit fly (Diptera: Tephritidae) larvae. Fla Entomol. 100, 743-751. doi: 10.1653/024.100.0422

Salomone, R., Saija, G., Mondello, G., Giannetto, A., Fasulo, S., and Savastano, D. (2017). Environmental impact of food waste bioconversion by insects: application of life cycle assessment to process using Hermetia illucens. J. Clean Prod. 140, 890-905. doi: 10.1016/j.jclepro.2016.06.154

Schloss, P. D., Westcott, S. L., Ryabin, T., Hall, J. R., Hartmann, M., Hollister, E. B., et al. (2009). Introducing mothur: open-source, platform-independent, community-supported software for describing and comparing microbial communities. Appl. Environ. Microbiol. 75, 7537-7541. doi: 10.1128/AEM. 01541-09

Schlüter, O., Rumpold, B., Holzhauser, T., Roth, A., Vogel, R. F., Quasigroch, W., et al. (2017). Safety aspects of the production of foods and food ingredients from insects. Mol. Nutr. Food Res. 61:1600520. doi: 10.1002/mnfr.20160 0520

Segata, N., Izard, J., Waldron, L., Gevers, D., Miropolsky, L., Garrett, W. S., et al. (2011). Metagenomic biomarker discovery and explanation. Genome Biol. 12:R60. doi: 10.1186/gb-2011-12-6-r60

Sharp, R. G. (2013). A review of the applications of chitin and its derivatives in agriculture to modify plant-microbial interactions and improve crop yields. Agronomy 3, 757-793. doi: 10.3390/agronomy3040757

Sheppard, D. C., Newton, G. L., Thompson, S. A., and Savage, S. (1994). A value added manure management system using the black soldier fly. Bioresour. Technol. 50, 275-279. doi: 10.1016/0960-8524(94)90102-3

Silvennoinen, K., Heikkilä, L., Katajajuuri, J.-M., and Reinikainen, A. (2015). Food waste volume and origin: case studies in the Finnish food service sector. Waste Manag. 46, 140-145. doi: 10.1016/j.wasman.2015.09.010

Somroo, A. A., ur Rehman, K., Zheng, L., Cai, M., Xiao, X., Hu, S., et al. (2019). Influence of Lactobacillus buchneri on soybean curd residue co-conversion by black soldier fly larvae (Hermetia illucens) for food and feedstock production. Waste Manag. 86, 114-122. doi: 10.1016/j.wasman.2019.01.022

Song, J., Kim, S.-Y., Kim, D.-H., Lee, Y.-S., Sim, J.-S., Hahn, B.-S., et al. (2018). Characterization of an inhibitor-resistant endo-1,4- $\beta$-mannanase from the gut microflora metagenome of Hermetia illucens. Biotechnol. Lett. 40, 1377-1387. doi: 10.1007/s10529-018-2596-2
Steinert, G., Wemheuer, B., Janussen, D., Erpenbeck, D., Daniel, R., Simon, M., et al. (2019). Prokaryotic Diversity and Community Patterns in Antarctic Continental Shelf Sponges. Front. Mar. Sci. 6:297. doi: 10.3389/fmars.2019. 00297

Taverner, P. D. (2002). "Drowning not waving? a perspective on the ways petroleum-derived oils kill arthropod pests of plants," in Spray Oils Beyond 2000: Sustainable Pest and Disease Management Spray Oils Beyond 2000: Sustainable Pest and Disease Management, ed. A. Beattie (Sydney, N.S.W: University of Western Sydney), 78-88.

Trinder, M., Bisanz, J. E., Burton, J. P., and Reid, G. (2015). Probiotic lactobacilli: a potential prophylactic treatment for reducing pesticide absorption in humans and wildlife. Benef Microbes 6, 841-847. doi: 10.3920/BM2015.0022

Verma, M., van den, B., Vreede, L., de, Achterbosch, T., and Rutten, M. M. (2020). Consumers discard a lot more food than widely believed: estimates of global food waste using an energy gap approach and affluence elasticity of food waste. PLoS One 15:e0228369. doi: 10.1371/journal.pone.0228369

Vogel, H., Müller, A., Heckel, D. G., Gutzeit, H., and Vilcinskas, A. (2018). Nutritional immunology: diversification and diet-dependent expression of antimicrobial peptides in the black soldier fly Hermetia illucens. Dev. Comp. Immunol. 78, 141-148. doi: 10.1016/j.dci.2017.09.008

Wagner, A. O., Markt, R., Puempel, T., Illmer, P., Insam, H., and Ebner, C. (2017). Sample preparation, preservation, and storage for volatile fatty acid quantification in biogas plants. Eng. Life Sci. 17, 132-139. doi: 10.1002/elsc. 201600095

Waldbauer, G. P. (1968). The consumption and utilization of food by insects. $A d v$. Insect Phys. 5, 229-288. doi: 10.1016/S0065-2806(08)60230-1

Wang, Y., and Rozen, D. E. (2018). Gut microbiota in the burying beetle, Nicrophorus vespilloides, provide colonization resistance against larval bacterial pathogens. Ecol. Evol. 8, 1646-1654. doi: 10.1002/ece3.3589

Wang, Y., Liu, H., and Sun, Z. (2017). Lamarck rises from his grave: parental environment-induced epigenetic inheritance in model organisms and humans. Biol. Rev. Camb. Philos. Soc. 92, 2084-2111. doi: 10.1111/brv.12322

Wemheuer, F., Taylor, J. A., Daniel, R., Johnston, E., Meinicke, P., Thomas, T., et al. (2020). Tax4Fun2: prediction of habitat-specific functional profiles and functional redundancy based on 16S rRNA gene sequences. Environ. Microbiome 15:11. doi: 10.1186/s40793-020-00358-7

Wickham, H. (2016). ggplot2: Elegant Graphics for Data Analysis. New York, NY: Springer.

Wong, C.-Y., Rosli, S.-S., Uemura, Y., Ho, Y. C., Leejeerajumnean, A., Kiatkittipong, W., et al. (2019). Potential protein and biodiesel sources from black soldier fly larvae: insights of larval harvesting instar and fermented feeding medium. Energies 12:1570. doi: 10.3390/en12081570

Wu, S., Xu, S., Chen, X., Sun, H., Hu, M., Bai, Z., et al. (2018). Bacterial Communities Changes during Food Waste Spoilage. Sci. Rep. 8:8220. doi: 10. 1038/s41598-018-26494-2

Wynants, E., Frooninckx, L., Crauwels, S., Verreth, C., De Smet, J., Sandrock, C., et al. (2018). Assessing the microbiota of black soldier fly larvae (Hermetia illucens) reared on organic waste streams on four different locations at laboratory and large scale. Microb. Ecol. 77, 913-930. doi: 10.1007/s00248-0181286-x

Zhan, S., Fang, G., Cai, M., Kou, Z., Xu, J., Cao, Y., et al. (2019). Genomic landscape and genetic manipulation of the black soldier fly Hermetia illucens, a natural waste recycler. Cell Res. 30, 50-60. doi: 10.1038/s41422-019-0252-6

Conflict of Interest: The authors declare that the research was conducted in the absence of any commercial or financial relationships that could be construed as a potential conflict of interest.

Copyright (C) 2021 Klammsteiner, Walter, Bogataj, Heussler, Stres, Steiner, SchlickSteiner and Insam. This is an open-access article distributed under the terms of the Creative Commons Attribution License (CC BY). The use, distribution or reproduction in other forums is permitted, provided the original author(s) and the copyright owner(s) are credited and that the original publication in this journal is cited, in accordance with accepted academic practice. No use, distribution or reproduction is permitted which does not comply with these terms. 